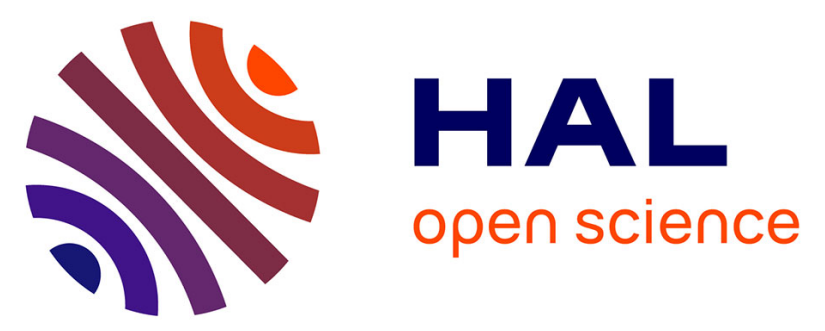

\title{
Electroosmotic Coupling in Porous Media, a New Model Based on a Fractal Upscaling Procedure
}

\author{
Luong Duy Thanh, Damien Jougnot, Phan van Do, Aida Mendieta, Nguyen \\ Xuan Ca, Vu Xuan Hoa, Pham Minh Tan, Nguyen Thi Hien
}

\section{- To cite this version:}

Luong Duy Thanh, Damien Jougnot, Phan van Do, Aida Mendieta, Nguyen Xuan Ca, et al.. Electroosmotic Coupling in Porous Media, a New Model Based on a Fractal Upscaling Procedure. Transport in Porous Media, 2020, 134 (1), pp.249-274. 10.1007/s11242-020-01444-7 . hal-02950764

\section{HAL Id: hal-02950764 https://hal.sorbonne-universite.fr/hal-02950764}

Submitted on 30 Sep 2020

HAL is a multi-disciplinary open access archive for the deposit and dissemination of scientific research documents, whether they are published or not. The documents may come from teaching and research institutions in France or abroad, or from public or private research centers.
L'archive ouverte pluridisciplinaire HAL, est destinée au dépôt et à la diffusion de documents scientifiques de niveau recherche, publiés ou non, émanant des établissements d'enseignement et de recherche français ou étrangers, des laboratoires publics ou privés. 


\title{
1. Electroosmotic coupling in porous media, a new model 2 based on a fractal upscaling procedure
}

\author{
$3 \quad$ Luong Duy Thanha, Damien Jougnot ${ }^{\mathrm{b}}$, Phan Van Doa, Aida Mendietab, \\ ${ }_{4}$ Nguyen Xuan $\mathrm{Ca}^{c}$, Vu Xuan Hoac, Pham Minh Tand, Nguyen Thi Hiene,f,* \\ aThuyloi University, 175 Tay Son, Dong Da, Hanoi, Vietnam \\ bSorbonne Universit'e, CNRS, EPHE, UMR 7619 Metis, F-75005, Paris, France \\ cFaculty of Physics and Technology, TNU-University of Sciences, Thai Nguyen, Vietnam \\ dFaculty of Fundamental Sciences, Thai Nguyen University of Technology, Thai Nguyen, \\ 11
}

9

10

12

${ }_{13}$ Abstract

Electrokinetic and electroosmotic couplings can play important roles in water and ions transport in charged porous media. Electroosmosis is the phenomena explaining the water movement in a porous medium subjected to an electrical field. In this work, a new model is obtained through a new up-scaling procedure, considering the porous medium as a bundle of tortuous capillaries of fractal nature. From the model, the expressions for the electroosmosis pressure coefficient, the relative electroosmosis pressure coefficient, the maximum back pressure, the maximum flow rate, the flow rate-applied back pressure relation and the product of the permeability and formation factor of porous media are also obtained. The sensitivity of the relative electroosmosis pressure coefficient is then analyzed and explained. The model predictions are then successfully compared with published datasets. Additionally, we deduce an expression for the relative streaming potential coefficient and then compare it with a previously published model and experimental data from a dolomite rock sample. We find a good agreement between those models and experimental 
data, opening up new perspectives to model electroosmotic phenomena in porous media saturated with various fluids. containing charged particles. It arises due to the induced movement of a $\operatorname{liq}_{20}$ uid by a voltage across a porous sample and is directly linked to an electrical ${ }_{21}$ double layer between the solid grain surface and the pore solution. Electroos ${ }_{22}$ mosis has been studied both experimentally and theoretically for a long time 2 (e.g., Reuss, 1809; Quincke, 1861; Smoluchowski, 1902; Nourbehecht, 1963; 24 Lyklema, 1995). Electroosmotic flow is a critical phenomenon that is used in 25 a variety of applications. For example, electroosmotic flow pumps have been 26 used in different fields of microfluidics such as biological and chemical anal ${ }_{27} y$ sis (Good et al., 2006), liquid drug reagent injection/delivery (e.g., Tsai \& ${ }_{28}$ Sue, 2007; Wang et al., 2009), microelectronic chip cooling (e.g., Linan Jiang ${ }_{29}$ et al., 2002; Singhal et al., 2004) and microfluidic devices (e.g., Hu \& Li, 2007; зо Bruus, 2008; Kirby, 2010). Solutes and nonaqueous phase liquids/dense non ${ }_{31}$ aqueous phase liquids can be removed by an electroosmotic technique in the ${ }_{2}$ vadose zone for remediation purposes (e.g., Bruell et al., 1992; Wise \& Tran 33 tolo, 1994; Han et al., 2004; Reddy et al., 1997). Additionally, electroosmo ${ }_{34}$ Sis has great potential for the dewatering of earth masonry structures (e.g., ${ }_{35}$ Casagrande, 1983; Lockhart \& Hart, 1988; Larue et al., 2006) or drying mois ${ }_{36}$ ture ingress in existing buildings, stone and earth masonry structures (e.g., ${ }_{37}$ Lockhart \& Hart, 1988; Ottosen \& Ro"rig-Dalgaard, 2006; Bertolini et al., 38 2009).

39 Many studies on electroosmosis flow reported in the literature use cylin40 drical capillaries or microchannels between two parallel plates (e.g., Rice ${ }_{41} \&$ Whitehead, 1965; Levine et al., 1975; Olivares et al., 1980; Ohshima \& ${ }_{42}$ Kondo, 1990; Mohiuddin Mala et al., 1997; Vennela et al., 2011). In 
porous ${ }_{43}$ media, electroosmotic flow has been presented using capillary bundle models ${ }_{44}$ with different capillary geometry such as rectangular, cylindrical and annular ${ }_{45}$ geometries (e.g., Wu \& Papadopoulos, 2000; Pascal et al., 2012). Bandopad 46 hyay et al. (2013) introduced the parameter of the electro-permeability that ${ }_{47}$ relates the flow rate with the applied voltage in porous media. Based on ${ }_{48}$ the electroosmotic flow in a single capillary, models for the height difference ${ }_{4}$ between the Utube experiment caused by electroosmosis in a fully saturated ${ }_{50}$ porous medium were presented (e.g., Paillat et al., 2000; Liang et al., 2015). ${ }_{51}$ For characterization of electroosmotic miropumps fabricated by packing non $_{52}$ porous silica particles, a bundle of capillary tubes model was applied (e.g., ${ }_{5}$ Zeng et al., 2001; Yao \& Santiago, 2003). Besides capillary tubes models, ${ }_{54}$ other approaches based on volume-averaging upscaling can be also applied ${ }_{55}$ to calculate the electrokinetic coupling in porous media (e.g., Pride, 1994; ${ }_{5}$ Revil \& Linde, 2006; Revil et al., 2007).

${ }_{57}$ It has been shown that natural porous media have fractal properties. ${ }_{58}$ Their pore space is statistically self-similar over several length scales (among ${ }_{9}$ many others, see Mandelbrot, 1982; Katz \& Thompson, 1985; Yu \& Cheng, ${ }_{0} 2002$ ). Theory on the fractal nature of porous media has attracted much ${ }_{61}$ attention in different areas (e.g., Mandelbrot, 1982; Feder \& Aharony, 1989). 62 Therefore, models based on the fractal theory have been applied to study ${ }_{3}$ phenomena in both fully and partially saturated porous media (e.g., Cai ${ }_{64}$ et al., 2012a,b; Liang et al., 2014; Guarracino \& Jougnot, 2018; Soldi et al., ${ }_{65}$ 2019; Thanh et al., 2018, 2019). However, to the best of our knowledge, ${ }_{6}$ the fractal theory has not yet been used to study electroosmosis in a porous ${ }_{67}$ medium under water saturated and partially saturated conditions.

${ }_{68}$ In this work, we apply fractal theory in porous media to obtain a mech ${ }_{69}$ anistic analytical model to describe electroosmotic flow in porous media us 70 ing a capillary tube model. From the derived model, the expressions for ${ }_{71}$ the electroosmotic pressure coefficient, the relative electroosmosis pressure 72 coefficient, the maximum back pressure, the maximum flow rate, the flow 73 rate-applied back pressure relation and the product of the formation fac ${ }_{74}$ tor and permeability of porous media are also obtained. To validate the 75 model, the sensitivity of the relative electroosmosis pressure coefficient $K_{E}{ }^{r}$

76 to irreducible water saturation $S_{i r r}$, ratio of the minimum pore radius to the 77 maximum pore radius $\alpha$ and fractal dimension for pore space $D_{f}$ is analyzed ${ }_{78}$ and explained. The model is then compared with published results in both 79 
cases of full saturation and partial saturation. Additionally, the expression 80 for the relative streaming potential coefficient $K_{S}{ }^{r}$ is also deduced from $K_{E}{ }^{r}$. ${ }_{81}$ From that, the change of the relative streaming potential coefficient $K_{S}^{r}$ with ${ }_{82}$ the water saturation is predicted and compared with another model and with ${ }_{83}$ experimental data for a dolomile rock sample available in literature.

\section{${ }_{84} 2$. Theoretical background of electroosmosis}

\section{2.1. Theory of electroosmosis}

${ }_{86}$ Porous media are constituted by minerals (e.g., silicates, oxides, carbon ${ }_{87}$ ates) or other materials (e.g., polymers, biological materials) that are gener ${ }_{88}$ ally electrically charged due to isomorphic substitutions (e.g., Hunter, 1981;

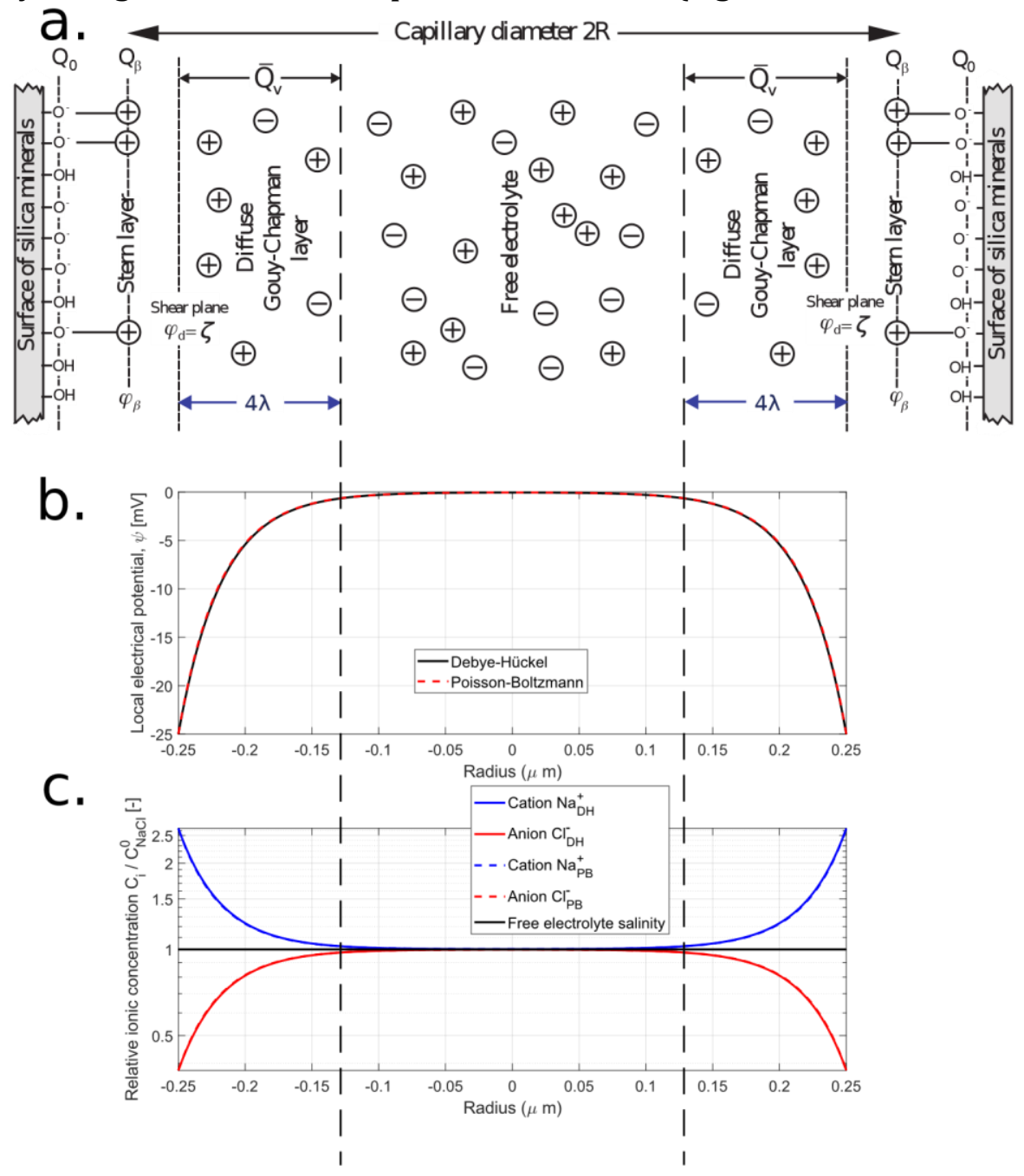


Figure 1: (a) Sketch of the electrical double layer at the surface of a mineral in contact with water. Comparison between the Debye-Hu"ckel (DH) approximation (plain line, Eq. (13)) and the Poisson-Boltzman equation (dashed line) to compute (b) the electrical potential distribution and (c) the ionic species relative concentration distribution in a capillary $(R=$ $0.25 \mu \mathrm{m}$ ) containing a $\mathrm{NaCl}$ electrolyte with $10^{-4} \mathrm{~mol} / \mathrm{L}$ (i.e., $\lambda=0.0304 \mu \mathrm{m}$ ). Note that the dashed and plain lines are perfectly superimposed, validating the use of Eq. (13).

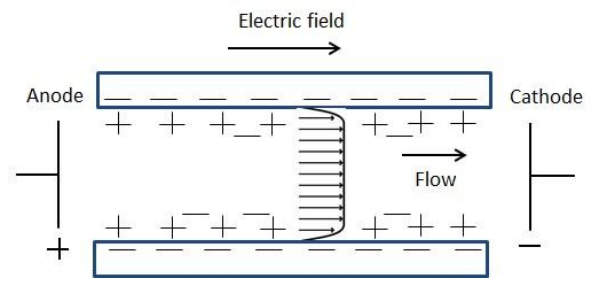

Figure 2: Electroosmosis flow in a capillary tube.

${ }_{89}$ Jacob \& Subirm, 2006) as shown in Fig. 1. The pore fluid nearby solid ${ }_{90}$ solution interface contains an excess of charges (counter-ions) to insure the 91 electro-neutrality of the entire system. These counter-ions are often cations $9_{2}$ and surface charges are often negatively charged in environmental conditions. 93 Note that the surface charges strongly depend on the $\mathrm{pH}$ and that the sign ${ }_{94} \mathrm{can}$ change at low $\mathrm{pH}$. The value at which the sign changes is called the point ${ }_{95}$ of zero charge (e.g., Hunter, 1981; Leroy \& Revil, 2004). This gives rise to ${ }_{96}$ the charge distribution known as the electrical double layer (EDL) as shown gin Fig. 1a. The EDL is composed of a Stern layer, where counter-ions are 98 adsorbed onto the solid surface and are immobile, and a diffuse layer that 99 contains mobile counter-ions and co-ions. In the diffuse layer, the distribu $u_{100}$ tion of ions and electric potential are governed by the Poisson-Boltzman (PB) ${ }_{101}$ equation in quasistatic conditions. The solution to the linear PB equation ${ }_{102}$ for a cylinder is well-known and the electric potential decays over distance ${ }_{103}$ from the charged surface as displayed in Fig. 1b using the code provided by ${ }_{10}$ Leroy \& Maineult (2018). Further away from the solid-solution is the bulk ${ }_{105}$ electrolyte, free from surface charge influence, it contains an equal number ${ }_{106}$ of cations and anions and is therefore electrically neutral (Fig. 1c). The ${ }_{107}$ shear plane or the slipping plane is the closest place to the solid in which ${ }_{108}$ water flow occurs and the electrical potential at this plane is called the zeta ${ }_{109}$ potential ( $\left.\zeta\right)$. The zeta potential depends on parameters including mineral ${ }_{110}$ composition of porous media, ionic species that are present in the fluid, the ${ }_{111} \mathrm{pH}$ of the fluid, fluid electrical conductivity and temperature etc. (see Hunter ${ }_{112}$ (1981); Davis et al. (1978); Jaafar et al. (2009) for more details). ${ }_{113}$ Reuss (1809) carried out the 
first experiment on electroosmosis by ap ${ }_{114}$ plying a DC voltage across a water saturated porous sample in a U-tube. ${ }_{115}$ When a DC voltage is applied across a capillary containing water, ions in the ${ }_{116} \mathrm{EDL}$ are submitted to an electric force and move to the electrode of oppo-

${ }_{117} \quad$ site polarity. That leads to the movement of the fluid near the solid surface ${ }_{118}$ as well as the bulk liquid due to viscous forces. The net motion of liquid is 119 called electroosmotic flow (Fig. 2). The pressure necessary to counterbalance ${ }_{120}$ electroosmotic flow is defined as the electroosmotic pressure (e.g., Jacob \& ${ }_{121}$ Subirm, 2006).

\section{2.2. Governing equations}

${ }_{123}$ The electrokinetic coupling in a fluid saturated porous medium is de ${ }_{124}$ Scribed by two linear equations (e.g., Li et al., 1995; Pengra et al., 1999)

$$
\begin{gathered}
\mathbf{U}_{e}=-\sigma \nabla V-\Pi_{12} \nabla P . \\
\mathbf{U}^{f}=-\Pi_{21} \nabla V-\frac{k}{\eta} \nabla P,
\end{gathered}
$$

${ }_{125}$ where $\mathbf{U}_{e}$ and $\mathbf{U}_{f}$ are the electric current density $\left(\mathrm{A} \mathrm{m}^{-2}\right)$ and Darcy flux ${ }_{126}(\mathrm{~m}$ $\mathrm{s}^{-1}$ ), $V$ is the electrical potential (V), $P$ is the pressure that drives the ${ }_{127}$ flow, $\sigma$ and $k$ are the electrical conductivity $\left(\mathrm{S} \mathrm{m}^{-1}\right)$ and permeability $\left(\mathrm{m}^{2}\right){ }_{128}$ of the porous medium, $\eta$ is the dynamic viscosity of the fluid, the off-diagonal 129 coefficients $\left(\Pi_{12}\right.$ and $\left.\Pi_{21}\right)$ are the electrokinetic coupling coefficients. In the

130 steady state, those coupling coefficients must satisfy the reciprocal relation

131 of Onsager: $\Pi_{12}=\Pi_{21}=\Pi$.

${ }_{132}$ The streaming potential coefficient is defined when the electric current ${ }_{133}$ density $\mathbf{U}_{e}$ is zero (e.g., Li et al., 1995; Wang et al., 2016), leading to

$$
K_{S}=\frac{\Delta V}{\Delta P}=-\frac{\Pi}{\sigma} .
$$

${ }_{134}$ Note that another formulation in which streaming potential coefficient for ${ }_{135}$ saturated porous media is described through the effective excess charge den ${ }_{136}$ sity $\widehat{Q}_{v}\left(\mathrm{C} / \mathrm{m}^{3}\right)$ dragged by the flow of the pore water was proposed by (e.g., ${ }_{137}$ Revil \& Leroy, 2004; Revil \& Linde, 2006)

$$
K_{S}=-\frac{\widehat{Q}_{v} k}{\sigma \eta} .
$$



$\mathbf{U}_{f}$ is zero (e.g., Li et al., 1995; Wang et al., 2016), leading to

$$
K_{E}=\frac{\Delta P}{\Delta V}=-\frac{\Pi \eta}{k}
$$

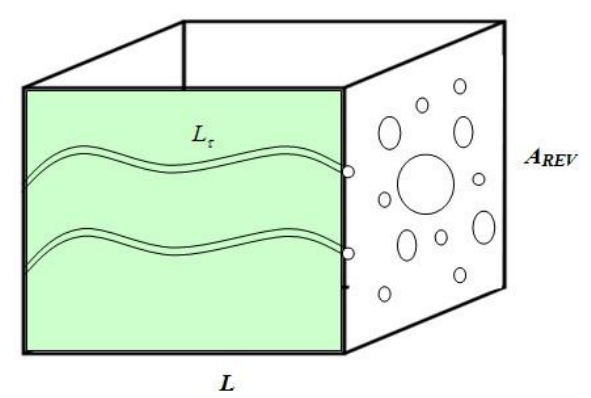

Figure 3: Sketch of the considered conceptual representative elementary volume (REV): Parallel and tortuous capillary tubes with radii following a fractal distribution.

By the volume averaging approach, Pride (1994) obtained the steady state ${ }_{141}$ coupling coefficient under a thin electrical double layer assumption as

$$
\Pi=\frac{\phi}{\tau} \frac{\epsilon_{r} \epsilon_{0} \zeta}{\eta}=\frac{\epsilon_{r} \epsilon_{0} \zeta}{F \eta},
$$

${ }_{142}$ where $r$ is the relative permittivity of the fluid, 0 is the dielectric per $_{143}$ mittivity in vacuum, $\varphi, \tau$ and $F$ are the porosity, hydraulic tortuosity and

\section{${ }_{151}$ 3. Model development}


${ }_{153}$ Consider binary symetric 1:1 electrolytes (e.g., $\mathrm{NaCl}$ ) of bulk ionic $\mathrm{con}_{154}$ centration $C^{0}\left(\mathrm{~mol} / \mathrm{m}^{3}\right)$ with an electrical potential $\psi(r)(\mathrm{V})$ at a distance ${ }_{155} r$ from the axis. If the excess charge density at this point is $\rho(r)\left(\mathrm{C} \mathrm{m}^{-3}\right),{ }_{156}$ then the Poisson equation is given by (e.g., Rice \& Whitehead, 1965; Gierst, 157 1966)

$$
\frac{1}{r} \frac{d}{d r}\left(r \frac{d \psi(r)}{d r}\right)=-\frac{\rho(r)}{\epsilon_{r} \epsilon_{0}},
$$

161 If $\left|\frac{e \psi(r)}{k_{b} T}\right|<<_{1}$ Pride,

or Poisson $_{163}$ now becomes

where $k_{b}$ is the Boltzmann's constant, $T$ is temperature (in K), $N$ is the ${ }_{160}$ Avogadro number and $e$ is the elementary charge.

1994; Hunter, 1981; Jougnot et al., 2019), $\sinh \frac{e \psi(r)}{k_{b} T} \approx \frac{e \psi(r)}{k_{b} T}$. The Boltzmann equation

$$
\begin{gathered}
\frac{1}{r} \frac{d}{d r}\left(r \frac{d \psi(r)}{d r}\right)=-\frac{2 N e^{2} C^{0}}{\epsilon_{0} \epsilon_{r} k_{b} T} \psi(r \\
\frac{1}{r} \frac{d}{d r}\left(r \frac{d \psi(r)}{d r}\right)=-\frac{\psi(r)}{\lambda^{2}}
\end{gathered}
$$

165 where $^{\lambda}=\sqrt{\frac{\epsilon_{0} \epsilon_{r} k_{b} T}{2 N e^{2} C^{0}}}$ is defined as the Debye length (e.g., Israelachvili, 1992). ${ }_{166}$ The boundary conditions of Eq. (11) to be satisfied for the cylindrical ${ }_{167}$ capillary surface are (Rice \& Whitehead, 1965):

$$
\psi(r)=\left\{\begin{array}{l}
\psi(R)=\zeta \\
\left.\frac{d \psi(r)}{d r}\right|_{r=0}=0
\end{array}\right.
$$

168 Under the boundary conditions given by Eq. (12), the analytical solution of $_{169} \quad \psi(r)$ and $\rho(r)$ are obtained as (Rice \& Whitehead, 1965)

$$
\psi(r)=\zeta \frac{I_{o}\left(\frac{r}{\lambda}\right)}{I_{o}\left(\frac{R}{\lambda}\right)}
$$


and

$$
\rho(r)=-\frac{\epsilon_{0} \epsilon \zeta}{\lambda^{2}} \frac{I_{o}\left(\frac{r}{\lambda}\right)}{I_{o}\left(\frac{R}{\lambda}\right)}
$$

where $I_{o}$ is the zero-order modified Bessel function of the first kind. Figure 1 compares the potential and concentrations of ions in the EDL calculated from ${ }_{173}$ Eq. (13) and the exact Poisson-Boltzmann solution (see Leroy \& Maineult ${ }_{174}(2018)$ for more details). It shows that Eq. (13) is a correct approximation ${ }_{175}$ for the Poisson-Boltzmann true solution.

\subsection{Velocity distribution at pore scale}

Under application of an electric field $E$ and a fluid pressure difference $\Delta P$ across a tortuous capillary of radius $R$, the fluid flow is the sum of a Poiseuille ${ }_{179}$ flow generated by $\Delta P$ and an electroosmotic flow generated by $E$ acting on ${ }_{180}$ the charge density in the EDL given by Eq. (14). Consequently, the velocity ${ }_{181}$ profile $v(r)$ in a cylindrical capillary is given as (Rice \& Whitehead, 1965)

$$
v(r)=\frac{1}{4 \eta}\left(R^{2}-r^{2}\right) \frac{\Delta P}{L_{\tau}}+\frac{\epsilon_{r} \epsilon_{0} \zeta E}{\eta}\left[1-\frac{I_{0}(r / \lambda)}{I_{0}(R / \lambda)}\right],
$$

where $L_{\tau}$ is the length of tortuous capillaries.

Because the electric field $E$ is related to the applied voltage across the ${ }_{184}$ porous medium $\Delta V$ by $E=\Delta V / L$ ( $L$ is the length of the porous medium as ${ }_{185}$ shown in Fig. 3). Eq. (15) is rewritten as

$$
v(r)=\frac{1}{4 \eta}\left(R^{2}-r^{2}\right) \frac{\Delta P}{L_{\tau}}+\frac{\epsilon_{r} \epsilon_{0} \zeta}{\eta}\left[1-\frac{I_{0}(r / \lambda)}{I_{0}(R / \lambda)}\right] \frac{\Delta V}{L} .
$$

The volume flow rate in the capillary is

$$
q(R)=\int_{0}^{R} v(r) 2 \pi r d r=\frac{\pi R^{4}}{8 \eta} \frac{\Delta P}{L_{\tau}}+\frac{\pi \epsilon_{r} \epsilon_{0} \zeta R^{2}}{\eta}\left[1-\frac{2 \lambda I_{1}(R / \lambda)}{R I_{0}(R / \lambda)}\right] \frac{\Delta V}{L},
$$

where $I_{1}$ is the first-order modified Bessel functions of the first kind.

Figure 4 shows the variation of the nondimensional parameter of the $\sec _{189}$ ond term in square brackets in Eq. (17) denoted by $C=2 \lambda I_{1}(R / \lambda) /\left(R I_{0}(R / \lambda)\right)$ with $R / \lambda$. It is seen that when the pore size is much bigger than the Debye length (hundred times), the term of $2 \lambda I_{1}(R / \lambda) /\left(R I_{0}(R / \lambda)\right)$ is much smaller than the unity and can be ignored (see Rice \& Whitehead (1965) for more 
details). Under that condition called the thin EDL assumption, Eq. (17) ${ }_{194}$ becomes

$$
q(R)=\frac{\pi R^{4} \Delta P}{8 \eta L_{\tau}}+\frac{\pi \epsilon_{r} \epsilon_{0} \zeta R^{2}}{\eta} \frac{\Delta V}{L} .
$$

In geological media and under most environmental conditions (i.e., ground-

water for human consumption or subsurface reservoirs), ionic strengths (i.e.,

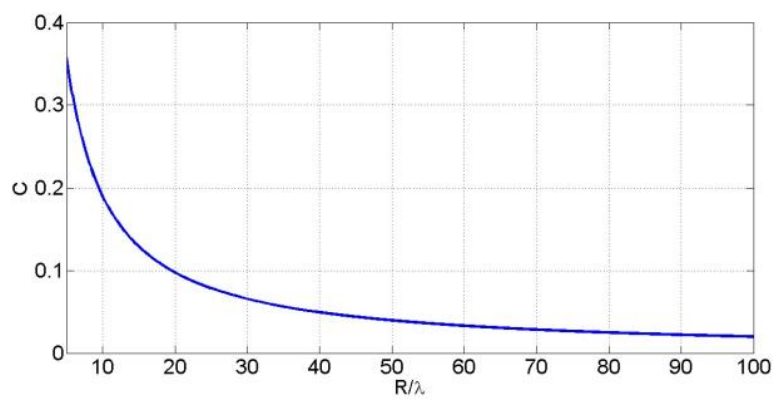

Figure 4: The variation of the unitless coefficient $\mathrm{C}=2 \lambda I_{1}(R / \lambda) /\left(R I_{0}(R / \lambda)\right)$ with the unitless ratio of the considered pore size and the Debye length $(R / \lambda)$

a proxy for ionic concentration) in potable water typically vary between $10^{-3}$ and $10^{-2} \mathrm{~mol} / \mathrm{L}$ (Jougnot et al., 2019). Reservoirs can be saturated with brine having much higher ionic concentrations depending on the for $_{200}$ mation. Therefore, the Debye length is typically less than $10 \mathrm{~nm}$ at $25^{\circ} \mathrm{C} 201$ (Israelachvili, 1992). It suggests that the minimum pore radius of porous ma ${ }_{202}$ terials that is applicable for thin EDL under the environmental conditions ${ }_{203}$ is around $100 \times 10 \mathrm{~nm}=1 \mu \mathrm{m}$. In addition, typical characteristic radius of ${ }_{204}$ pore in geological media is tens of micrometer (e.g., Hu et al., 2017). There 205 fore, a thin EDL assumption (no EDL overlap) is normally satisfied in most ${ }_{206}$ natural systems (see Jougnot et al. (2019) for more details). It is noted that ${ }_{207}$ the thin EDL assumption does not work for clay rocks and low permeability 208 sediments where the pore size is on the order of $10 \mathrm{~nm}$. Therefore, one needs ${ }_{209}$ to take into account the term of $C=2 \lambda I_{1}(R / \lambda) /\left(R I_{0}(R / \lambda)\right)$ in Eq. (17). It 210 is therefore a limitation to the proposed model. 


\section{3.3. Fractal based up-scaling}

${ }_{212}$ Porous media can be conceptualized as a bundle of tortuous capillary ${ }_{213}$ tubes following a fractal pore-size distribution (e.g., Yu \& Cheng, 2002; Liang ${ }_{214}$ et al., 2014) (see Fig. 3). The fractal approach is a simple and elegant way to ${ }_{215}$ upscale microscopic properties to macroscopic properties by assuming that ${ }_{216}$ the pore size distribution follows the fractal scaling law

$$
N(\geq R)=\left(\frac{R_{\max }}{R}\right)^{D_{f}},
$$

${ }_{217}$ where $N$ is the number of capillaries with radius greater than $R, R_{\max }$ is the ${ }_{218}$ maximum radius, $D_{f}$ is the fractal dimension for pore space, $0<D_{f}<2{ }_{219} \quad$ in two-dimensional space and $0<D_{f}<3$ in three dimensional space ( $\mathrm{Yu} \& 220$ Cheng, 2002; Liang et al., 2014).

From Eq. (19), the following is obtained

$$
-d N=D_{f} R_{\max }^{D_{f}} R^{-D_{f}-1} d R,
$$

where $-d N$ is the number of capillaries with radius ranging from $R$ to $R+d R$. ${ }_{223}$ The minus (-) in Eq. (20) implies that the number of pores decreases with 224 the increase of pore size. The total number of capillaries with radius ranging 225 from $R_{\min }$ (the minimum radius) to $R_{\max }$ (the maximum radius) is given by

$$
N_{\text {total }}\left(\geq R_{\text {min }}\right)=\left(\frac{R_{\max }}{R_{\min }}\right)^{D_{f}} .
$$

From Eq. (20) and Eq. (21), the following is obtained

$$
-\frac{d N}{N_{\text {total }}}=D_{f} R_{\min }^{D_{f}} R^{-D_{f}-1} d R=f(R) d R,
$$

in which $f(R)=D_{f} R_{\min }^{D_{f}} R^{-D_{f}-1}$ is the probability density function. According to the statistical theory, the probability density function needs to meet ${ }_{229}$ the following condition

$$
\int_{R_{\min }}^{R_{\max }} f(R) d R=1-\left(\frac{R_{\min }}{R_{\max }}\right)^{D_{f}}=1
$$

or 


$$
\left(\frac{R_{\min }}{R_{\max }}\right)^{D_{f}} \approx 0
$$

231 Eq. (24) is approximately valid for $R_{\min } / R_{\max } \approx 10^{-2}$ or $<10^{-2}$

(Yu \& ${ }_{232}$ Cheng, 2002; Liang et al., 2014). That condition generally holds in porous ${ }_{233}$ media. The fractal dimension $D_{f}$

is linked to the porosity of porous media ${ }_{234}$ and the ratio of

the minimum capillary radius to the maximum capillary ${ }_{235}$ radius $\left(\alpha=R_{\min } / R_{\max }\right.$ ) by following equation (e.g., Yu et al., 2001; Yu \& ${ }_{236}$ Cheng, 2002)

$$
D_{f}=2-\frac{\ln \phi}{\ln \alpha}
$$

237 3.4. REV scale

${ }_{238}$ To obtain the volume flow rate at the macroscale, a representative ele ${ }_{239}$ mentary volume (REV) as a cube with a length $L$ is considered. As presented ${ }_{240}$ in the previous section, the porous medium exhibits a fractal pore size dis ${ }_{241}$ tribution with radii varying from $R_{\min }$ to $R_{\max }$. We consider the REV under ${ }_{242}$ varying saturation conditions. The effective saturation is defined by

$$
S_{e}=\frac{S_{w}-S_{i r r}}{1-S_{i r r}},
$$

${ }_{248}$ where $T_{S}(\mathrm{~N} / \mathrm{m})$ is the surface tension of the fluid and $\beta$ is the contact angle. ${ }_{249}$ A capillary becomes fully desaturated under the pressure head $h$ if $R$ is ${ }_{250}$ greater than the radius $R_{h}$ given by Eq. (27). Therefore, the capillaries with 251 radii $R$ between $R_{\min }$ and $R_{h}$ will be fully saturated under the pressure head

where $S_{w}$ is the water saturation and $S_{i r r}$ is irreducible water saturation.

We assume that the REV is initially fully saturated and then drained ${ }_{245}$ when submitted to a pressure head $h(\mathrm{~m})$. For a capillary tube, the pore ${ }_{246}$ radius $R_{h}(\mathrm{~m})$ that is drained at a given pressure head $h$ can be calculated ${ }_{247}$ by (Jurin, 1719)

$$
h=\frac{2 T_{s} \cos \beta}{\rho_{w} g R_{h}},
$$

$h$.

For porous media containing only large and regular pores, the irreducible ${ }_{254}$ water saturation can often be neglected.

For porous media containing small ${ }_{255}$ pores, the irreducible water saturation can be pretty significant because water ${ }_{256}$ 
remains trapped in the crevices or in micropores that are not occupied by air. 257 This amount of water is taken into account in the model by setting irreducible 258 water radius of capillaries $R_{\text {irr }}$. Consequently, the following assumptions are

${ }_{259}$ made in this work: (1) for $R_{\min } \leq R \leq R_{i r}$, the capillaries are occupied by 260 water that is immobile at irreducible saturation due to insufficient driving ${ }_{261}$ force, so it does not contribute to fluid flow; (2) for $R_{i r r}<R \leq R_{h}$, the ${ }_{262}$ capillaries are occupied by mobile water, so it contributes to the fluid flow; ${ }_{263}$ (3) for $R_{h}<R$ $\leq R_{\max }$, the capillaries are occupied by air, so it does not ${ }_{264}$ contribute to the fluid flow. In this work, film bound water adhering to 265 the capillary walls of porous media with radius greater than $R_{\text {irr }}$ is ignored. ${ }_{266}$ Therefore, the irreducible water saturation is defined as

$$
S_{i r r}=\frac{\int_{R_{\min }}^{R_{i r r}} \pi R^{2} L_{\tau}(-d N)}{\int_{R_{\min }}^{R_{\max }} \pi R^{2} L_{\tau}(-d N)}=\frac{R_{i r r}^{2-D_{f}}-R_{\min }^{2-D_{f}}}{R_{\max }^{2-D_{f}}-R_{\min }^{2-D_{f}}} .
$$

The water saturation is determined as:

$$
S_{w}=\frac{\int_{R_{\min }}^{R_{h}} \pi R^{2} L_{\tau}(-d N)}{\int_{R_{\min }}^{R_{\max }} \pi R^{2} L_{\tau}(-d N)}=\frac{R_{h}^{2-D_{f}}-R_{\min }^{2-D_{f}}}{R_{\max }^{2-D_{f}}-R_{\min }^{2-D_{f}}} .
$$

Because only capillaries with radius between $R_{\min }$ and $R_{h}$ are fully saturated ${ }_{269}$ under a pressure head $h$, the volumetric flow rate $Q_{R E V}$ through the REV ${ }_{270}$ is the sum of the flow rates over all capillaries with radius between $R_{i r r}$ and ${ }_{271} R_{h}$ and given by

$$
Q_{R E V}=\int_{R_{\text {irr }}}^{R_{h}} q(R)(-d N)
$$

272 Combining Eq. (18), Eq. (20) and Eq. (30), the following is obtained

$$
Q_{R E V}=\frac{\pi R_{\max }^{D_{f}}}{8 \eta} \frac{D_{f}\left(R_{h}^{4-D_{f}}-R_{i r r}^{4-D_{f}}\right)}{\left(4-D_{f}\right)} \frac{\Delta P}{L_{\tau}}+\frac{\pi R_{\max }^{D_{f}} \epsilon_{r} \epsilon_{0} \zeta}{\eta} \frac{D_{f}\left(R_{h}^{2-D_{f}}-R_{\text {irr }}^{2-D_{f}}\right)}{\left(2-D_{f}\right)} \frac{\Delta V}{L}
$$

Ad3 Aditionally, from Eq. (28) and Eq. (29) one has

$$
R_{i r r}=R_{\max }\left[\alpha^{2-D_{f}}+S_{i r r}\left(1-\alpha^{2-D_{f}}\right)\right]^{\frac{1}{2-D_{f}}}
$$

and 


$$
R_{h}=R_{\max }\left[\alpha^{2-D_{f}}+S_{w}\left(1-\alpha^{2-D_{f}}\right)\right]^{\frac{1}{2-D_{f}}},
$$

275 where $\alpha=R_{\min } / R_{\max }$.

276 Combining Eq. (31), Eq. (32) and Eq. (33), the following is obtained

$$
\begin{aligned}
Q_{R E V}= & \frac{\pi R_{\max }^{4} D_{f}}{8 \eta\left(4-D_{f}\right)}\left\{\left[\alpha^{2-D_{f}}+S_{w}\left(1-\alpha^{2-D_{f}}\right)\right]^{\frac{4-D_{f}}{2-D_{f}}}-\left[\alpha^{2-D_{f}}+S_{i r r}\left(1-\alpha^{2-D_{f}}\right)\right]^{\frac{4-D_{f}}{2-D_{f}}}\right\} \frac{\Delta P}{L_{\tau}} \\
& +\frac{\pi R_{\max }^{2} \epsilon_{r} \epsilon_{0} \zeta D_{f}}{\eta\left(2-D_{f}\right)} S_{w}\left(1-\alpha^{2-D_{f}}\right)\left(S_{w}-S_{i r r}\right) \frac{\Delta V}{L} .
\end{aligned}
$$

where $\tau$ is the mean tortuosity of the porous medium defined by the relation ${ }_{283} L_{\tau}=\tau$.L.

The cross sectional area of the REV is therefore obtained as

$$
A_{R E V}=\frac{\pi \tau D_{f} R_{\max }^{2}\left(1-\alpha^{2-D_{f}}\right)}{\left(2-D_{f}\right) \phi} .
$$

Combining Eq. (34), Eq. (35) and Eq. (37), one obtains

$$
\begin{aligned}
Q= & \frac{R_{\max }^{2} A}{8 \eta \tau} \frac{\left(2-D_{f}\right)}{\left(4-D_{f}\right)} \frac{\phi}{\left(1-\alpha^{2-D_{f}}\right)} \times \\
& \left\{\left[\alpha^{2-D_{f}}+S_{w}\left(1-\alpha^{2-D_{f}}\right)\right]^{\frac{4-D_{f}}{2-D_{f}}}-\left[\alpha^{2-D_{f}}+S_{i r r}\left(1-\alpha^{2-D_{f}}\right)\right]^{\frac{4-D_{f}}{2-D_{f}}}\right\} \frac{\Delta P}{L_{\tau}} \\
& +\frac{\epsilon_{r} \epsilon_{0} \zeta \phi A}{\eta \tau}\left(S_{w}-S_{i r r}\right) \frac{\Delta V}{L}
\end{aligned}
$$

where $A$ and $A_{R E V}$ are the cross sectional areas of the porous medium and ${ }_{279}$ the REV, both are perpendicular to the flow direction.

The porosity is calculated by

$$
\phi=\frac{V_{\text {pore }}}{V_{R E V}}=\frac{\int_{R_{\min }}^{R_{\max }} L_{\tau} \pi R^{2}(-d N)}{L A_{R E V}}=\frac{\pi \tau D_{f} R_{\max }^{2}\left(1-\alpha^{2-D_{f}}\right)}{\left(2-D_{f}\right) A_{R E V}},
$$

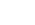


${ }_{304}$ Under the condition of zero back pressure difference $(\Delta P=0)$, the total 305 flow rate is maximum as

$$
Q_{m}=\frac{\epsilon_{r} \epsilon_{0} \zeta A \phi}{\eta \tau}\left(S_{w}-S_{i r r}\right) \frac{\Delta V}{L}
$$


${ }_{306}$ Combining Eq. (38), Eq. (41) and Eq. (42), the link between the pressure ${ }_{307}$ difference and the flow rate is given by

$$
Q=Q_{m}\left[1-\frac{\Delta P}{\Delta P_{m}}\right]
$$

${ }_{308}$ Eq. (43) is exactly the same as that obtained in Zeng et al. (2001) in which ${ }_{309}$ the porous medium was conceptualized as a bundle of parallel capillaries of ${ }_{310}$ the same radii at full saturation condition. Interestingly, Eq. (43) is obtained 311 in this work for the fractal pore size distribution and for partially saturated ${ }_{312}$ porous media but the result is the same for the relationship between flow ${ }_{313}$ rate and pressure difference.

314 3.6. Electroosmotic pressure coefficient

${ }_{315}$ The electroosmotic pressure coefficient $K_{E}$ is defined by $\Delta P / \Delta V$, that ${ }_{316}$ means the $K_{E}$ is a macroscopic variable (i.e., integrating over the entire ${ }_{317}$ bundle of capillaries) when the total flow rate $Q$ in Eq. (38) equals zero ( $\mathrm{Li}_{318}$ et al., 1995; Wang et al., 2016). Consequently, one has

$$
\begin{aligned}
K_{E}= & \left.\frac{\Delta P}{\Delta V}\right|_{Q=0}=\frac{\Delta P_{m}}{\Delta V} \quad(44)_{319} \text { Using Eq. (41), Eq. (44) is rewritten as } \\
K_{E}= & -\frac{8 \epsilon_{r} \epsilon_{0} \zeta \tau(1-\phi)\left(4-D_{f}\right)}{R_{\max }^{2}\left(2-D_{f}\right)} \times \\
& \frac{S_{w}-S_{i r r}}{\left\{\left[\alpha^{2-D_{f}}+S_{w}\left(1-\alpha^{2-D_{f}}\right)\right]^{\frac{4-D_{f}}{2-D_{f}}}-\left[\alpha^{2-D_{f}}+S_{i r r}\left(1-\alpha^{2-D_{f}}\right)\right]^{\frac{4-D_{f}}{2-D_{f}}}\right\}} .
\end{aligned}
$$

Eq. (45) is a general expression for the electroosmotic pressure coefficient

for partially saturated porous media. Eq. (45) indicates that the electroos ${ }_{322}$ motic pressure coefficient is explicitly linked to $\zeta$, microstructural parameters ${ }_{323}$ of porous media $\left(D_{f}, \varphi, \alpha, R_{\max }, \tau\right)$, water saturation and irreducible water ${ }_{324}$ saturation. Therefore, the model can indicate more mechanisms influencing ${ }_{325}$ the electroosmotic pressure coefficient than other published models (e.g., Eq.

(7) deduced by the volume averaging approach).

In case of full saturation $S_{w}=1$, Eq. (45) becomes 


$$
K_{E}^{s a t}=-\frac{8 \epsilon_{r} \epsilon_{0} \zeta \tau(1-\phi)\left(4-D_{f}\right)}{R_{\max }^{2}\left(2-D_{f}\right)} \frac{1-S_{i r r}}{\left\{1-\left[\alpha^{2-D_{f}}+S_{i r r}\left(1-\alpha^{2-D_{f}}\right)\right]^{\frac{4-D_{f}}{2-D_{f}}}\right\}}
$$

328

$$
\begin{aligned}
& K_{E}^{r}= \frac{K_{E}}{K_{E}^{\text {sat }}}=\frac{S_{w}-S_{i r r}}{1-S_{\text {irr }}} \frac{\left\{1-\left[\alpha^{2-D_{f}}+S_{i r r}\left(1-\alpha^{2-D_{f}}\right)\right]^{\frac{4-D_{f}}{2-D_{f}}}\right\}}{\left\{\left[\alpha^{2-D_{f}}+S_{w}\left(1-\alpha^{2-D_{f}}\right)\right]^{\frac{4-D_{f}}{2-D_{f}}}-\left[\alpha^{2-D_{f}}+S_{i r r}\left(1-\alpha^{2-D_{f}}\right)\right]^{\frac{4-D_{f}}{2-D_{f}}}\right\}} \\
&=S_{e} \frac{\left\{1-\left[\alpha^{2-D_{f}}+S_{i r r}\left(1-\alpha^{2-D_{f}}\right)\right]^{\frac{4-D_{f}}{2-D_{f}}}\right\}}{\left\{\left[\alpha^{2-D_{f}}+S_{w}\left(1-\alpha^{2-D_{f}}\right)\right]^{\frac{4-D_{f}}{2-D_{f}}}-\left[\alpha^{2-D_{f}}+S_{i r r}\left(1-\alpha^{2-D_{f}}\right)\right]^{\frac{4-D_{f}}{2-D_{f}}}\right\}} .
\end{aligned}
$$

\section{Results and discussion}

4.1. Predictions of the model under partially saturated conditions

${ }_{331}$ The values of $\alpha$ between 0.001 and 0.01 are used in this section for model $_{332}$ ing because those values are normally used in published works (Yu \& Cheng, ${ }_{33}$ 2002; Liang et al., 2014; Thanh et al., 2019). The fractal dimension $D_{f}$ is in ${ }_{334}$ the range between 1 and 2. For given porous media, $D_{f}$ is determined via ${ }_{335} \mathrm{Eq}$. (25) with known values of $\alpha$ and porosity $\varphi$. Fig. 5 shows (a) the influ ${ }_{336}$ ence of the irreducible saturation on the change of the relative electroosmotic ${ }_{337}$ pressure coefficient $K_{E}^{r}$ with the water saturation $S_{w}\left(S_{i r r}=0.02,0.05\right.$ and

0.1 ) for $\alpha=0.01$ and $D_{f}=1.8$; (b) influence of $\alpha$ on the variation of $K_{E}^{r}$ with $S_{w}(\alpha=0.002,0.005,0.01)$ for $S_{i r r}=0.05$ and $D_{f}=1.8$ and (c) Influ ${ }_{340}$ ence of $D_{f}$ on the variation of $K_{E}{ }^{r}$ with $S_{w}\left(D_{f}=1.3,1.5,1.8\right)$ for $\alpha=0.01{ }_{341}$ and $S_{\text {irr }}=$ 0.05 . The results show that as the media desaturate, the relative ${ }_{342}$ electroosmotic pressure coefficient $K_{E}^{r}$ increases. The reason is that at lower ${ }_{343}$ water saturation, only capillaries with smaller radii are occupied by water. ${ }_{344}$ Therefore, one needs larger pressure differences over porous media to counter ${ }_{345}$ balance with the flow rate generated by the same applied voltage (such that ${ }_{346}$ the total volumetric flow rate is zero). As a result, $K_{E} r$ is larger for lower 
${ }_{347}$ water saturation. Additionally, Fig. 5 shows that the relative electroosmotic ${ }_{348}$ pressure coefficient is very sensitive to the fractal dimension $D_{f}$ and the ratio ${ }_{349}$ $\alpha$. It is noted that the $D_{f}$ decreases with an increase of $\alpha$ at a given porosity ${ }_{350}$ as indicated by Eq. (25). Fig. 5 also shows that the $K_{E^{r}}$ decreases with the ${ }_{351}$ increase of the ratio $\alpha$ and decrease of $D_{f}$. That can be explained by the ${ }_{352}$ decrease of the total number of pores in the REV with the decrease of $D_{f}$.

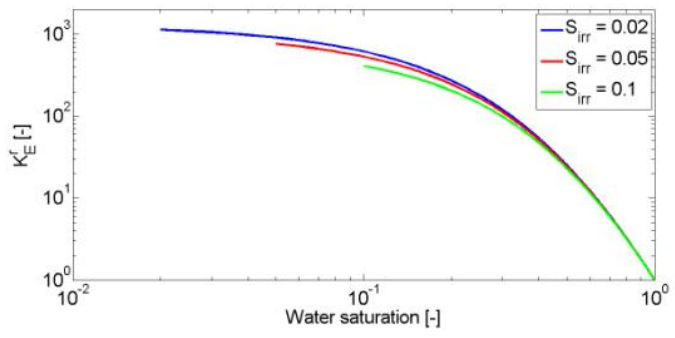

(a)

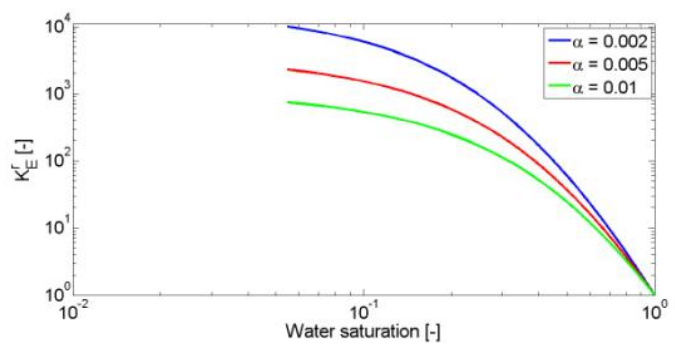

(b)

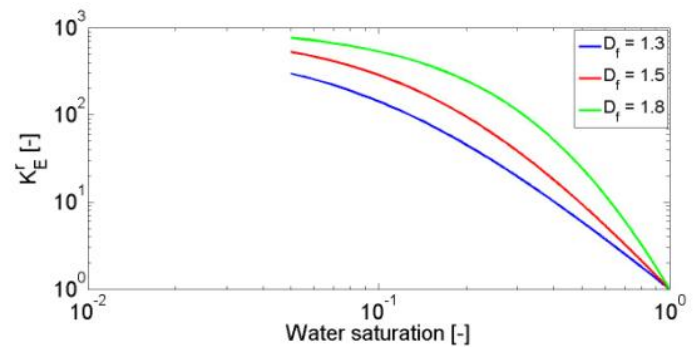

(c)

Figure 5: Sensitivity analysis of the model: (a) Influence of the irreducible saturation on the variation of the $K_{E}^{r}$ with the water saturation $S_{w}\left(S_{i r r}=0.02,0.05\right.$ and 0.1$)$ for $\alpha=$ 0.01 and $D_{f}=1.8$; (b) Influence of $\alpha$ on the variation of $K_{E}^{r}$ with $S_{w}(\alpha=0.002,0.005$, 0.01 ) for $S_{i r r}=0.05$ and $D_{f}=1.8$; (c) Influence of $D_{f}$ on the variation of $K_{E}{ }^{r}$ with $S_{w}\left(D_{f}=1.3\right.$, $1.5,1.8$ ) for $\alpha=0.01$ and $S_{\text {irr }}=0.05$. 


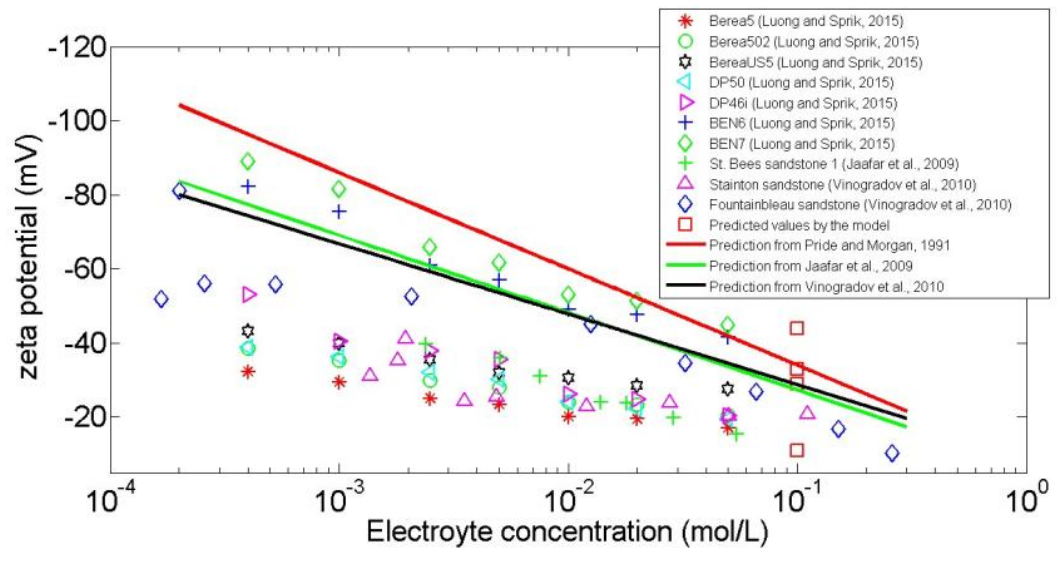

Figure 6: The variation of the zeta potential with pore fluid salinity. The predicted values of the zeta potential for four samples of glass beads at $0.1 \mathrm{~mol} / \mathrm{L} \mathrm{in} \mathrm{Li} \mathrm{et} \mathrm{al.} \mathrm{(1995)} \mathrm{are} \mathrm{shown}$ by red squares. Experimental data from different sources reported by Thanh \& Sprik (2015),Jaafar et al. (2009) and Vinogradov et al. (2010) are also presented.

${ }_{353}$ Therefore, $R_{h}$ becomes larger at the same water water saturation. Similarly, 354 we need to apply a smaller pressure difference over the partially saturated ${ }_{355}$ $\mathrm{REV}$ to counter balance the flow rate generated by the same applied voltage. 356 Consequently, $K_{E}^{r}$ decreases with a decrease of $D_{f}$.

${ }_{357}$ 4.2. Comparison with experimental data in water saturated porous media

${ }_{358}$ Even if the present model is developed based on the concept of capillary ${ }_{359}$ tubes, it possible to provide a relationship between capillary radius to grain 360 size for the sake of medium characterization. Indeed, in non-consolidated ${ }_{361}$ granular materials, pore size are very difficult to obtain without perturbat${ }_{362}$ ing the medium, while grain sizes and grain size distribution can be easily ${ }_{363}$ measured. Therefore, with the knowledge of the mean grain diameter $d$ of ${ }_{364} \mathrm{a}$ granular material, the maximum pore radius can be determined by (e.g., 365 Liang et al., 2015)

$$
R_{\text {max }}=\frac{d}{4}\left[\sqrt{\frac{\phi}{1-\phi}}+\sqrt{\frac{1}{1-\phi}}\right] .
$$

${ }_{366}$ Mean geometrical tortuosity of porous media is predicted from porosity ${ }_{367}$ as (e.g., Cai et al., 2012a; Ghanbarian et al., 2013; Liang et al., 2015)

$$
\tau=\frac{1}{2}\left[1+\frac{1}{2} \sqrt{1-\phi}+\sqrt{1-\phi} \frac{\sqrt{(1 / \sqrt{1-\phi}-1)^{2}+1 / 4}}{1-\sqrt{1-\phi}}\right]
$$


Table 1: Input parameters of four samples of fused glass beads are taken from Li et al. (1995). Symbols of $d, \varphi, K_{E}$ and $\alpha$ are symbols for the grain diameter, porosity, the electroosmotic pressure coefficient and ratio of minimum and maximum radius, respectively. The values of $\zeta$ are predicted from Eq. (45)

\begin{tabular}{cccccc}
\hline Number & $d(\mu \mathrm{m})$ & $\varphi$ (no units) & $K_{E}(\mathrm{~Pa} / \mathrm{V})$ & $\alpha$ (no units) & $\zeta(\mathrm{mV})$ \\
\hline 1 & 50 & 0.10 & 4.94 & 0.01 & -11 \\
2 & 50 & 0.17 & 12.5 & 0.01 & -48 \\
3 & 100 & 0.19 & 1.9 & 0.01 & -33 \\
4 & 200 & 0.30 & 0.25 & 0.01 & -29 \\
\hline mean & & & & -30
\end{tabular}

${ }_{368}$ We want to see if the model is able to predict the zeta potential using Eq. ${ }_{369}$ (46). Li et al. (1995) measured $K_{E}$ for fused glass beads fully saturated by ${ }_{370} 0.1$ $\mathrm{M} \mathrm{NaCl}$ electrolyte. Micro-structure parameters of the samples such ${ }_{371}$ as grain diameter $d$, porosity $\varphi$ as well as the measured $K_{E}$ are reported in ${ }_{372} \mathrm{Li}$ et al. (1995) and re-shown in Table 1. At this electrolyte concentration, s3 $^{\text {the }}$ the Debye length $\lambda$ is around $1 \mathrm{~nm}$ (Israelachvili, 1992). Of all samples, 374the minimum value of $R_{\max }$ corresponding to the sample 1 ( $d=50 \mu \mathrm{m}$ and ${ }_{375} \varphi=0.1$ ) is predicted using Eq. (48) to be $17 \mu \mathrm{m}$. Hence, $R_{\min }$ corresponding

${ }_{376}$ to the sample 1 is obtained using $R_{\min }=\alpha R_{\max }=0.01 R_{\max }=170 \mathrm{~nm}$. 377 Consequently, the Debye length is much smaller than $R_{\min }$ and Eq. (46) is ${ }_{378}$ applicable for experimental data reported by Li et al. (1995).

${ }_{379}$ The value of $\alpha$ is taken as 0.01 because that is a normally used value ${ }_{380}$ for grain materials (e.g., Thanh et al., 2018, 2019). The value of $S_{\text {irris }}$ s81 reasonably taken as 0 for large grain materials (e.g., Jougnot et al., 2012). ${ }_{382}$ From the known values of porosity $\varphi$ and $\alpha$ of samples (see Table 1 ), $D_{f}$ is ${ }_{383}$ obtained by Eq. (25). From Eq. (46), the zeta potential is obtained with the ${ }_{384}$ knowledge of $\alpha, D_{f}, \tau, \varphi, R_{\max }$ and $S_{\text {irr. }}$ The predicted values are presented ${ }_{385}$ in Table 1. It is seen that the predicted values (see red squares at $0.1 \mathrm{~mol} / \mathrm{L}{ }_{386} \mathrm{in} \mathrm{Fig.} \mathrm{6)} \mathrm{are} \mathrm{in}$ good agreement with published experimental data (Jaafar ${ }_{387}$ et al., 2009; Vinogradov et al., 2010; Thanh \& Sprik, 2015).

${ }_{388}$ The zeta potential is dependent on the electrical conductivity of the fluid. 389 The electroosmotic pressure coefficient $K_{E}^{\text {sat }}$ varies with the electrolyte elec 390 trical conductivity $\sigma_{w}$. Fig. 7 shows the variation of the electroosmotic pres 391 sure coefficient with fluid electrical conductivity experimentally obtained by 


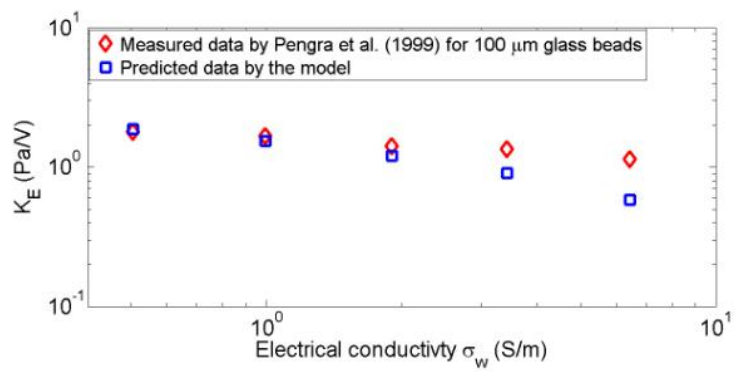

(a)

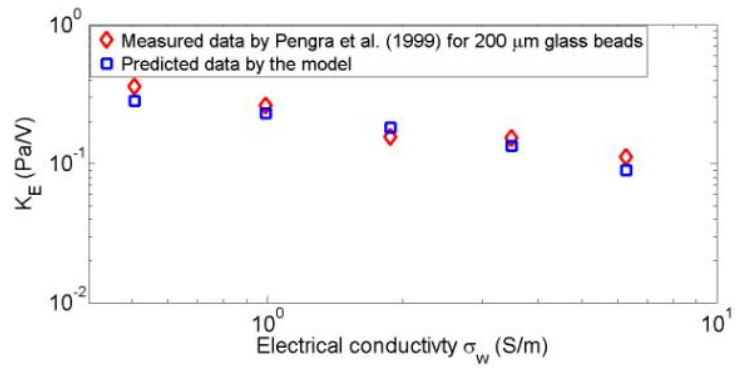

(b)

Figure 7: Variation of the electroosmotic pressure coefficient $K_{E}^{s a t}$ at saturated condition with electrolyte electrical conductivity for two consolidated samples of glass beads: (a) for the sample of $100 \mu \mathrm{m}$ glass beads and (b) for the sample of $200 \mu \mathrm{m}$ glass beads shown in Table 1).

${ }_{392}$ Pengra et al. (1999) for two samples of glass beads ( $d=100 \mu \mathrm{m}$ and $200 \mu \mathrm{m},{ }_{393}$ respectively) saturated by a $\mathrm{NaCl}$ electrolyte (see diamond symbols). There ${ }_{394}$ are few proposed expressions for the relationship between the zeta potential 395 and electrolyte concentration available in the literature (e.g., Pride \& Mor ${ }_{396}$ gan, 1991; Jaafar et al., 2009; Vinogradov et al., 2010). One is given by $\zeta={ }_{397} a+$ $b \log 10\left(C_{f}\right)$ with $a=-6.43 \mathrm{mV}, b=20.85 \mathrm{mV}$ as shown by Jaafar et al. ${ }_{398}(2009)$, for example. Electrical conductivity of the $\mathrm{NaCl}$ electrolyte is linked ${ }_{399}$ to the electrolyte concentration by the relation $\sigma_{w}=10 C_{f}$ for the ranges ${ }_{400} 10^{-6} \mathrm{M}<C_{f}<$ $1 \mathrm{M}$ and $15^{\circ} \mathrm{C}<T<25^{\circ} \mathrm{C}$ (Sen \& Goode, 1992). Therefore, ${ }_{401}$ the relation $\zeta=$ $-6.43+20.85 \log 10\left(0.1 \sigma_{w}\right)(\mathrm{mV})$ is obtained. Similarly, we ${ }_{402}$ obtain $R_{\min }=400$ $\mathrm{nm}$ for two samples and maximum value of $\lambda=1.36 \mathrm{~nm}{ }_{403}$ for the considered range of electrolyte concentration by Pengra et al. (1999) ${ }_{404}$ (from $0.05 \mathrm{~mol} / \mathrm{L}$ to $0.65 \mathrm{~mol} / \mathrm{L})$. Therefore, the thin EDL assumption is ${ }_{405}$ satisfied. Applying the same approach as we did previously, the variation ${ }_{406}$ of the electroosmotic pressure coefficient $K_{E}{ }^{\text {sat }}$ with electrical conductivity ${ }_{407} \sigma_{w}$ is predicted as shown in Fig. 7 by square symbols. One can see that ${ }_{408}$ the model prediction is also in 
good match with data measured by Pengra ${ }_{409}$ et al. (1999) (diamond symbols) even there is a large difference between the ${ }_{410}$ model prediction and measured data for smaller glass beads at high electrical ${ }_{411}$ conductivity. The reason may be that Jaafar et al. (2009) obtained:

$$
\zeta=a+b \log 10\left(C_{f}\right)
$$

${ }_{412}$ by fitting experimental data for quartz, silica, glass and St. Bees in $\mathrm{NaCl}_{413}$ brine with big data scattering. As shown in Fig. 3 of Jaafar et al. (2009), ${ }_{414}$ the difference in $\zeta$ can reach $65 \%$ at $C_{f}=10^{-2} \mathrm{~mol} / \mathrm{L}$. Therefore, the $\zeta-C_{f}$

415 relation may not work well for a single silica-based sample in a large range ${ }_{416}$ of electrolyte concentration. As a matter of fact, Cherubini et al. (2018) ${ }_{417}$ show that, for data on carbonate materials, the best fit they obtain is rather ${ }_{418} a=-6.97 \mathrm{mV}$ and $b=9.13 \mathrm{mV}$, indicating that this relationship is largely ${ }_{419}$ mineral dependent.

${ }_{420}$ Figure 8 shows the variation of $\Delta P_{m}$ with an applied voltage for the $10{ }_{421} \mu \mathrm{m}$ sand pack saturated with $10^{-3} \mathrm{M} \mathrm{NaCl}$. The symbols are deduced from ${ }_{422}$ Luong \& Sprik (2013) (their Fig. 10) using the relation $\Delta P_{m}=\rho g \Delta h_{m}\left(\Delta h_{m}\right.$ ${ }_{423}$ is the maximum height difference obtained from Luong \& Sprik (2013), $\rho$

$=$

Table 2: Parameters taken from Wang et al. (2015) for 10 sandstone samples in which $\varphi, k$, $\zeta$ stand for porosity, permeability, the zeta potential. The electroosmotic pressure coefficient $K_{E}^{e x p}$ is deduced by comparison between the similarity of porosity, permeability, depth of samples between Wang et al. (2015) and Wang et al. (2016). $K_{E}^{\text {theo }}$ is predicted from the model.

\begin{tabular}{cccccc}
\hline Sample & $\varphi$ (no units) & $k(10-15 \mathrm{~m} 2)$ & $\zeta(\mathrm{V})$ & KEexp $(\mathrm{Pa} / \mathrm{V})$ & KEtheo $(\mathrm{Pa} / \mathrm{V})$ \\
\hline D1 & 30.6 & 1028 & -0.0486 & 0.42 & 0.44 \\
D2 & 30.2 & 1435 & -0.0571 & 0.47 & 0.35 \\
D3 & 30.9 & 1307 & -0.0410 & 0.40 & 0.31 \\
D4 & 32.1 & 1152 & -0.0609 & 0.40 & 0.61 \\
D5 & 29.8 & 456 & -0.0727 & 0.52 & 1.30 \\
D6 & 31.0 & 978 & -0.0462 & 0.51 & 0.46 \\
D7 & 29.4 & 594 & -0.0627 & 0.49 & 0.82 \\
D8 & 31.0 & 2785 & -0.1448 & 0.52 & 0.51 \\
D9 & 29.3 & 1491 & -0.0765 & 0.43 & 0.40
\end{tabular}




$$
\begin{aligned}
& 4_{425} \begin{array}{l}
\text { D10 } \\
\text { gravity). At the saturated condition, Eq. (41) becomes }
\end{array} \\
& \qquad P_{m}=-\frac{8 \epsilon_{r} \epsilon_{0} \zeta \Delta V \tau(1-\phi)\left(4-D_{f}\right)}{R_{\max }^{2}\left(2-D_{f}\right)} \times \\
& \\
& \qquad 1-S_{\text {irr }} \\
& \left\{1-\left[\alpha^{2-D_{f}}+S_{\text {irr }}\left(1-\alpha^{2-D_{f}}\right)\right]^{\frac{4-D_{f}}{2-D_{f}}}\right\}
\end{aligned}
$$

426 The solid line is predicted from Eq. (51) in the same manner as mentioned ${ }_{427}$ above with $\varphi=0.38, D_{f}=0.01, d=10 \mu \mathrm{m}, \alpha=0.01$ and the mean value

${ }_{428}$ of $\zeta=-13 \mathrm{mV}$ over six granular samples made of spherical grains (Luong \& ${ }_{429}$ Sprik, 2013) (best fit is obtained with $S_{i r r}=0$ ). Note that the thin EDL is not ${ }_{430}$ really met in this case because of $R_{\min } \approx 60 \mathrm{~nm}$ and $\lambda=9.6 \mathrm{~nm}$. Therefore, ${ }_{431}$ the model may not work really well to reproduce the experimental data as 432 shown in Fig. 8.

${ }_{433}$ Eq. (46) is applied to determine the electroosmotic pressure ${ }^{434} K_{E}$ coefficient for ten sandstone samples $(20 \mathrm{~mm}$ in length and $25 \mathrm{~mm}$ in diameter) sat ${ }_{435}$ urated by a $0.05 \mathrm{M} \mathrm{NaCl}$ electrolyte reported in Wang et al. (2015). Parame ${ }_{436}$ ters of the sandstone samples and the measured zeta potential are presented

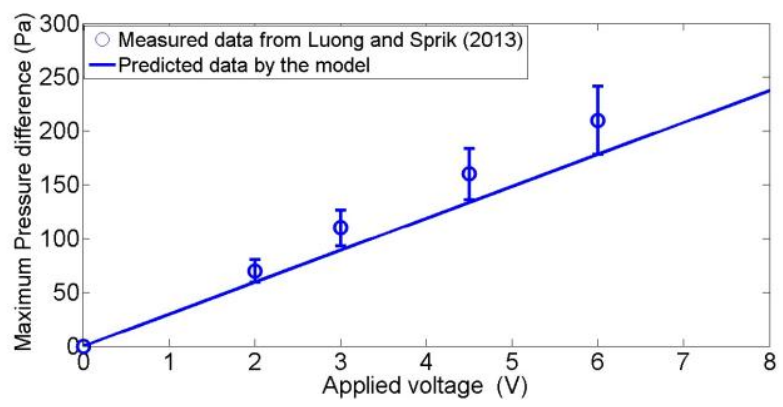

Figure 8: Maximum pressure difference as a function of applied voltage. The symbols are experimental data from Luong \& Sprik (2013) with $\pm 15 \%$ of uncertainty and the solid line is obtained from Eq. (51) 


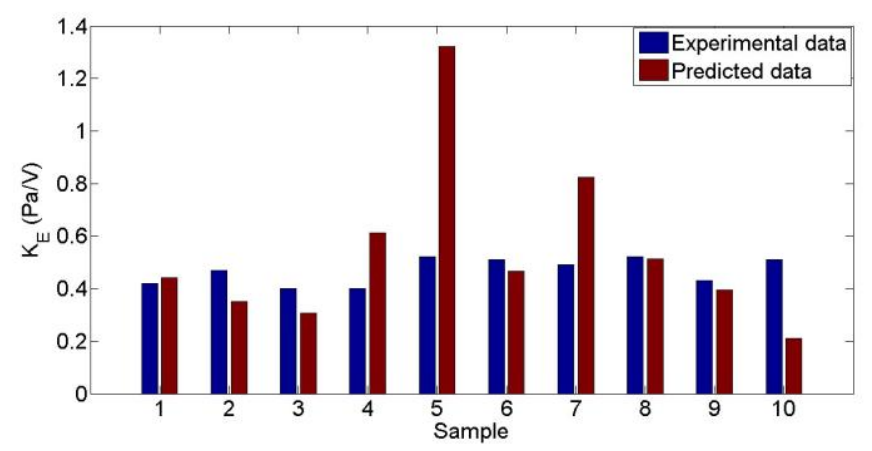

Figure 9: The variation of the $K_{E}$ predicted in this work and the experimental data taken from Wang et al. (2015)

${ }_{437}$ by Wang et al. (2015) and re-shown in Table 2 . The measured values of $K_{E 438}$ are obtained by the same research group Wang et al. (2016) for the same $\exp$

${ }_{439}$ conditions and re-presented in Table 2 (see $K_{E}$ ). To estimate the mean ${ }_{440}$ grain diameter of porous media from the permeability, we use the relation ${ }_{441}$ given by (e.g., Bernabe \& Revil, 1995; Revil et al., 1999; Glover et al., 2006)

$$
d=\sqrt{\frac{4 a m^{2} k}{\phi^{3 m}}}
$$

442 where the cementation exponent $m$ is taken as 1.9 for consolidated sandstone ${ }_{443}$ (Friedman, 2005) and $a$ is a constant between 2-12 that depends on the pore ${ }_{444}$ space (Glover et al., 2006; Glover \& Walker, 2009). In this part, $a$ is taken ${ }_{445}$ as 4 for a set of samples of Wang et al. (2015). With estimated values of ${ }_{446} d$, measured $\varphi$ and $\alpha=0.001$ (that value is also relevant to that used in ${ }_{447}$ Liang et al. (2014) for a Fontainebleau sandstone), the $K_{E}$ is predicted for ${ }_{448}$ reported in Table 2 ( $K_{E}$ theo $)$. Fig. 9 shows the predicted $K_{E}$ calculated in ${ }_{49}$ this work and measured values taken from Wang et al. (2015). It is seen that ${ }_{450} \mathrm{Eq}$. (46) predicts the published experimental data well. Note that for this ${ }_{451}$ set of experimental data, we obtain the minimum value of $R_{\min } \approx 2.10^{3} \mathrm{~nm}{ }_{452}$ and $\lambda=1.36 \mathrm{~nm}$. Therefore, the thin EDL assumption is satisfied and Eq. ${ }_{453}(46)$ is valid.

${ }_{454}$ 4.3. Prediction of the product of the permeability and formation factor ${ }_{455}$ Comparing Eq. (7) and Eq. (46), the product of the permeability and ${ }_{456}$ formation factor of porous media is given by 


$$
k F=\frac{R_{\max }^{2}\left(2-D_{f}\right)}{8 \tau(1-\phi)\left(4-D_{f}\right)} \frac{\left\{1-\left[\alpha^{2-D_{f}}+S_{i r r}\left(1-\alpha^{2-D_{f}}\right)\right]^{\frac{4-D_{f}}{2-D_{f}}}\right\}}{1-S_{i r r}}
$$

${ }_{457}$ Eq. (53) indicates that based on the fractal model for electroosmostic flow ${ }_{458}$ in porous media, one can get the product of the permeability and formation ${ }_{459}$ factor from the parameters $D_{f}, R_{\max }, \tau, \alpha$ and $S_{i r r}$ of porous media. Eq. (53) ${ }_{460}$ is now used to estimate the product of $k F$ and compare with experimental ${ }_{461}$ data reported in Glover et al. (2006), Glover \& D'ery (2010) and Bol'eve et al. ${ }_{462}$ (2007) for 27 samples of bead packs. Parameters for the samples (grain ${ }_{463}$ diameter, porosity, permeability) are taken from Glover et al. (2006), Glover ${ }_{464}$ \& D'ery (2010) and Bol`eve et al. (2007) and re-shown in Table 3. The values

Table 3: Input parameters for bead packs taken from Glover et al. (2006), Glover \& D'ery (2010) and Bol'eve et al. (2007). Symbols of $d(\mu \mathrm{m}), \varphi$ (no units), $k\left(\mathrm{~m}^{2}\right), F$ (no units) and $\alpha$ (no units) stand for grain diameter, porosity, permeability, formation factor and ratio of minimum and maximum radius, respectively.

\begin{tabular}{ccccccc}
\hline No. & $d(\mu \mathrm{m})$ & $\varphi(-)$ & $k(10-12 \mathrm{~m} 2)$ & $F(-)$ & $\alpha(-)$ & reference \\
\hline 1 & 20 & 0.40 & 0.2411 & 3.90 & 0.01 & Glover et al. (2006) \\
2 & 45 & 0.39 & 1.599 & 4.01 & 0.01 & Glover et al. (2006) \\
3 & 106 & 0.39 & 8.118 & 4.04 & 0.01 & Glover et al. (2006) \\
4 & 250 & 0.40 & 50.46 & 3.97 & 0.01 & Glover et al. (2006) \\
5 & 500 & 0.38 & 186.79 & 4.08 & 0.01 & Glover et al. (2006) \\
6 & 1000 & 0.40 & 709.85 & 3.91 & 0.01 & Glover et al. (2006) \\
7 & 2000 & 0.39 & 2277.26 & 4.13 & 0.01 & Glover et al. (2006) \\
8 & 3350 & 0.40 & 7706.97 & 3.93 & 0.01 & Glover et al. (2006) \\
\hline 9 & 1.05 & 0.411 & 0.00057 & 3.80 & 0.01 & Glover \& D'ery (2010) \\
10 & 2.11 & 0.398 & 0.00345 & 3.98 & 0.01 & Glover \& D'ery (2010) \\
11 & 5.01 & 0.380 & 0.0181 & 4.27 & 0.01 & Glover \& D'ery (2010) \\
12 & 11.2 & 0.401 & 0.0361 & 3.94 & 0.01 & Glover \& D'ery (2010) \\
13 & 21.5 & 0.383 & 0.228 & 4.22 & 0.01 & Glover \& D'ery (2010) \\
14 & 31 & 0.392 & 0.895 & 4.07 & 0.01 & Glover \& D'ery (2010) \\
15 & 47.5 & 0.403 & 1.258 & 3.91 & 0.01 & Glover \& D'ery (2010) \\
16 & 104 & 0.394 & 6.028 & 4.04 & 0.01 & Glover \& D'ery (2010) \\
17 & 181 & 0.396 & 21.53 & 4.01 & 0.01 & Glover \& D'ery (2010) \\
18 & 252 & 0.414 & 40.19 & 3.75 & 0.01 & Glover \& D'ery (2010) \\
19 & 494 & 0.379 & 224 & 4.29 & 0.01 & Glover \& D'ery (2010) \\
20 & 990 & 0.385 & 866.7 & 4.19 & 0.01 & Glover \& D'ery (2010) \\
\hline
\end{tabular}




\begin{tabular}{ccccccl}
\hline 21 & 56 & 0.4 & 2.0 & 3.3 & 0.01 & Bol’eve et al. (2007) \\
22 & 72 & 0.4 & 3.1 & 3.2 & 0.01 & Bol’eve et al. (2007) \\
23 & 93 & 0.4 & 4.4 & 3.4 & 0.01 & Bol'eve et al. (2007) \\
24 & 181 & 0.4 & 27 & 3.3 & 0.01 & Bol’eve et al. (2007) \\
25 & 256 & 0.4 & 56 & 3.4 & 0.01 & Bol’eve et al. (2007) \\
26 & 512 & 0.4 & 120 & 3.4 & 0.01 & Bol’eve et al. (2007) \\
27 & 3000 & 0.4 & 14000 & 3.6 & 0.01 & Bol’eve et al. (2007) \\
\hline
\end{tabular}

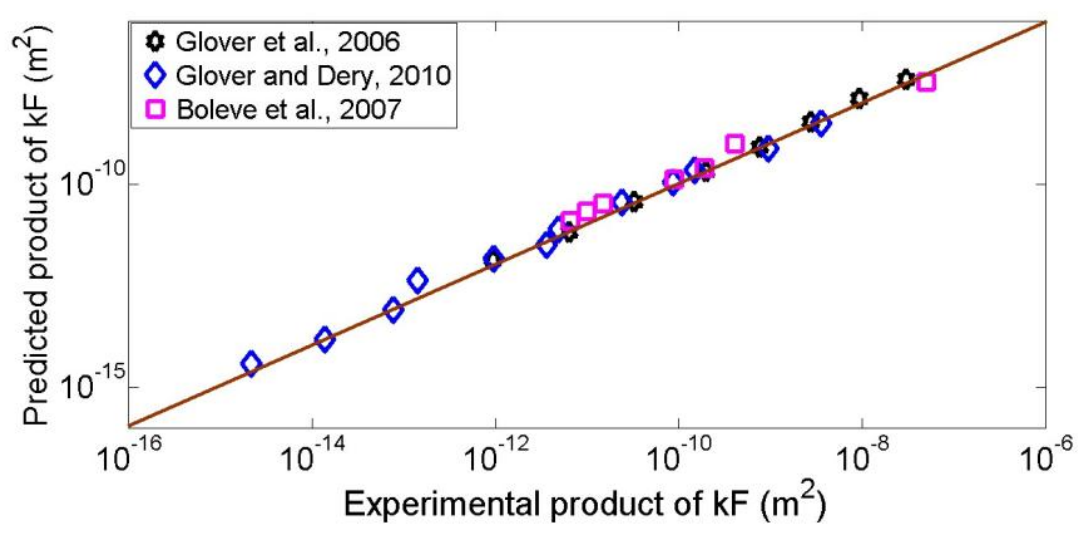

Figure 10: A comparison between $k F$ predicted from Eq. (53) and from measured data in Glover et al. (2006), Glover \& D'ery (2010) and Bol'eve et al. (2007) (the solid line is a 1:1 line).

${ }_{465}$ of $\alpha$ and $S_{\text {irr }}$ are taken as 0.01 and 0 , respectively. Value of $\tau$ is obtained by ${ }_{466}$ the relation $\tau=\varphi F$. From those parameters in combination with Eq. (53), 467 the product of the permeability and formation factor is predicted in the same ${ }_{468}$ procedure as previously mentioned. Fig. 10 shows the comparison between ${ }_{46}$ the product of $k F$ predicted in this work and the experimental data. The ${ }_{470}$ solid line represents a 1:1 line. It is seen that the predicted values are in very ${ }_{471}$ good match with the experimental data. It suggests that one can predict $k_{472}$ of porous materials from Eq. (53) with the knowledge of $F$ and vice versa.

473 4.4. Electrokinetic coupling under partially saturated condition

${ }_{474}$ Based on Eq. (3) and Eq. (5), the relationship between the electroosmosis ${ }_{475}$ coefficient and the streaming potential coefficient is obtained as

$$
K_{S}\left(S_{w}\right)=\frac{K_{E}\left(S_{w}\right) k\left(S_{w}\right)}{\sigma\left(S_{w}\right) \eta} .
$$


Therefore, the relative streaming potential coefficient is given as

$$
K_{S}^{r}=\frac{K_{S}\left(S_{w}\right)}{K_{S}^{s a t}\left(S_{w}=1\right)}=\frac{K_{E}\left(S_{w}\right)}{K_{E}^{s a t}\left(S_{w}=1\right)} \frac{k\left(S_{w}\right)}{k\left(S_{w}=1\right)} \frac{\sigma\left(S_{w}=1\right)}{\sigma\left(S_{w}\right)},
$$

where $K_{E}^{r}=\frac{K_{E}\left(S_{w}\right)}{K_{E}^{s a t}\left(S_{w}=1\right)}$ is given in Eq. (47), $\frac{k\left(S_{w}\right)}{k\left(S_{w}=1\right)}$ and $\frac{\sigma\left(S_{w}\right)}{\sigma\left(S_{w}=1\right)}$ are called the relative permeability and the relative conductivity of porous media and ${ }_{479}$ denoted by $k^{r}$ and $\sigma^{r}$, respectively. In this work, we do not have expressions 480 for $k^{r}$ and $\sigma^{r}$ based on the fractal theory yet. Therefore, we use expressions ${ }_{481}$ given by (e.g., Revil et al., 2007; Linde et al., 2006) for $k^{r}$ and $\sigma^{r}:$

$$
k^{r}=S_{e}^{(2+3 \lambda) / \lambda}
$$

and

$$
\sigma^{r}=S_{w}^{n}
$$

where is $\lambda$ is the curve-shape parameter and $n$ is the saturation exponent. ${ }_{884} \mathrm{Eq}$. (57) is valid for the negligible surface conductivity.

485 Combining Eq. (47), Eq. (55), Eq. (56) and Eq. (57), the relative

$$
\begin{aligned}
& \text { streaming potential coefficient is given by } \\
& K_{S}^{r}=S_{e} \frac{\left\{1-\left[\alpha^{2-D_{f}}+S_{i r r}\left(1-\alpha^{2-D_{f}}\right)\right]^{\frac{4-D_{f}}{2-D_{f}}}\right\}}{\left\{\left[\alpha^{2-D_{f}}+S_{w}\left(1-\alpha^{2-D_{f}}\right)\right]^{\frac{4-D_{f}}{2-D_{f}}}-\left[\alpha^{2-D_{f}}+S_{i r r}\left(1-\alpha^{2-D_{f}}\right)\right]^{\frac{4-D_{f}}{2-D_{f}}}\right\}} \\
& \quad \times S_{e}^{(2+3 \lambda) / \lambda} \frac{1}{S_{w}^{n}} .
\end{aligned}
$$

Additionally, Revil et al. (2007) used the volume averaging approach to get ${ }_{488}$ the relative streaming potential coefficient as

$$
K_{S}^{r}=S_{e}^{(2+3 \lambda) / \lambda} \frac{1}{S_{w}^{n+1}} .
$$

Figure 11 shows the change of the relative streaming potential coefficient $K_{S}{ }^{r}$ with the water saturation predicted from Eq. (58) and Eq. (59). Input ${ }_{991}$ parameters for modeling in Fig. 11 are $n=2.7, \lambda=0.87, S_{\text {irr }}=0.36$ which ${ }_{492}$ are 
reported by Revil et al. (2007), $D_{f}=1.5$ and $\alpha=0.01$ which are used ${ }_{493}$ due to the best fit. Additionally, a comparison between those models and ${ }_{494}$ experimental data reported in Revil et al. (2007) for the dolomite sample E3 ${ }_{495}$ (square symbols) is also shown in Fig. 11. A good agreement is observed ${ }_{496}$ between the proposed model, the model presented by Revil et al. (2007) 497 and experimental data. Clearly, both theoretical models are able to describe ${ }_{49}$ the decrease of the relative streaming potential coefficient with decreasing ${ }_{49}$ water saturation, from full saturation to nearly irreducible water saturation ${ }_{500}$ as indicated by experimental data. When $S_{w}$ decreases then the number of

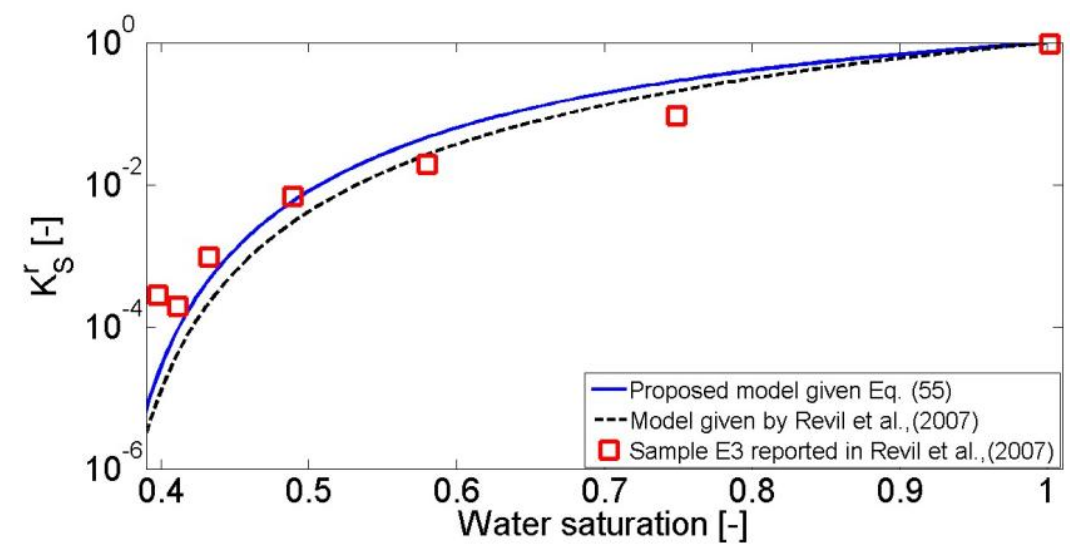

Figure 11: Variation of the coefficient $K_{S}^{r}$ with the water saturation $(n=2.7, \lambda=0.87$, $S_{\text {irr }}=0.36, D_{f}=1.5$ and $\alpha=0.01$ ). The solid and dashed lines correspond to the proposed model (see Eq. (58)) and the model of Revil et al. (2007), respectively.

${ }_{501}$ capillaries occupied by water in the REV decreases. Hence, the streaming ${ }_{502}$ current through the REV generated by a fluid flow becomes smaller at a given ${ }_{503}$ fluid pressure difference. Consequently, the $K_{S}{ }^{r}$ decreases with decreasing $S_{w 504}$ as predicted.

\subsection{Effective excess charge density}

From Eq. (3), Eq. (4), Eq. (5) and Eq. (46), the effective excess charge

density $\widehat{Q}_{v}\left(\mathrm{C} / \mathrm{m}^{3}\right)$ under fully saturated conditions is deduced as

$$
\widehat{Q}_{v}=\frac{8 \epsilon_{r} \epsilon_{0} \zeta \tau(1-\phi)\left(4-D_{f}\right)}{R_{\text {max }}^{2}\left(2-D_{f}\right)} \frac{1-S_{\text {irr }}}{\left\{1-\left[\alpha^{2-D_{f}}+S_{\text {irr }}\left(1-\alpha^{2-D_{f}}\right)\right]^{\frac{4-D_{f}}{2-D_{f}}}\right\}} \text {. }
$$

When one neglects the irreducible water saturation, Eq. (60) reduces to 


$$
\widehat{Q}_{v}=\frac{8 \epsilon_{r} \epsilon_{0} \zeta \tau(1-\phi)\left(4-D_{f}\right)}{R_{\max }^{2}\left(2-D_{f}\right)}
$$

509

${ }_{510}$ Based on Eq. (61), we can calculate $\widehat{Q}_{v}$ for the glass beads reported ${ }_{511}$ in Glover \& D'ery (2010) and Bol'eve et al. (2007) using the same approach ${ }_{512}$ mentioned above. The values of the zeta potential are reported to be $\zeta={ }_{513}$ $24.72 \mathrm{mV}$ and $-73.34 \mathrm{mV}$ in Glover \& D'ery (2010) and $\zeta=-17.5 \mathrm{mV},-44.7$

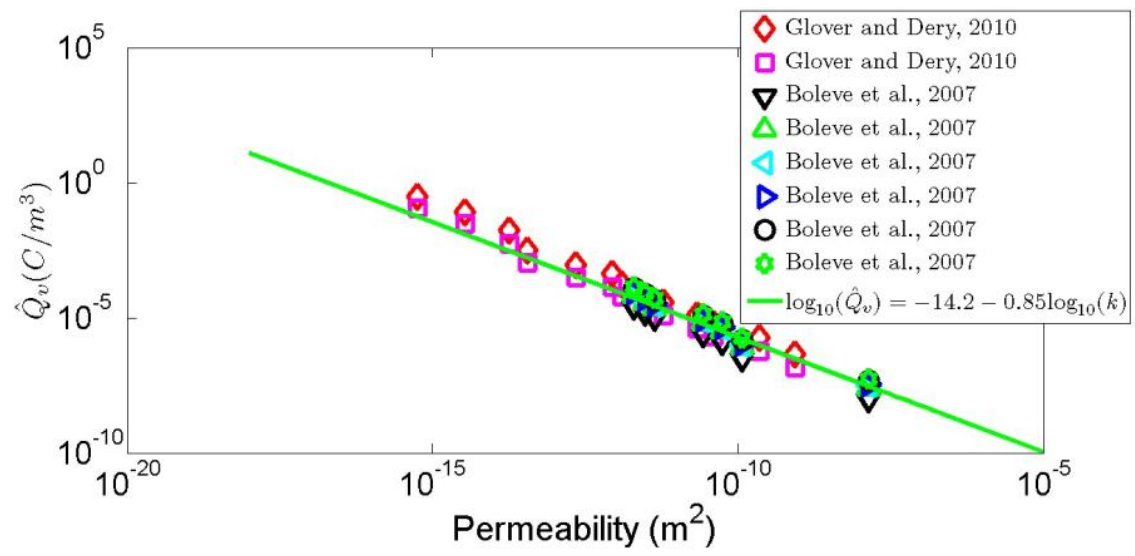

Figure 12: Variation of the effective excess charge density $Q_{\mathrm{b}_{v}}$ with the permeability $k$. Symbols represent experimental data from Glover \& D'ery (2010) and Bol’eve et al. (2007) (Table 3). The solid line is the fit line with $\log _{10}\left(\widehat{Q}_{v}\right)=-14.2-0.85 \log _{10}(k)$.

$\mathrm{mV},-54.6 \mathrm{mV},-59.7 \mathrm{mV},-87.9 \mathrm{mV}$ and $-99.3 \mathrm{mV}$ in Bol'eve et al. (2007) which $k$ is taken from Table 3) as shown in Fig. 12 from which we obtain

${ }_{517}$ the fit line: $\log _{10}(Q \mathrm{~b} v)=A_{1}+A_{2} \log _{10}(k)$ with $A_{1}=-14.2$ and $A_{2}=-0.85$. 518 The obtained $Q_{\mathrm{b} v}-k$ relationship is in good agreement with that reported

by Jardani et al. (2007) by fitting to a large set of experimental data that

includes various lithologies and ionic concentrations: $\log 10\left(\widehat{Q}_{v}\right)=-9.23-0.82$ $\log _{10}(k)$. The constant $A_{2}=-0.85$ obtained in this work is related to rock ${ }_{522}$ properties $\left(R_{\max }, \alpha, \tau, \varphi\right.$ and $\left.D_{f}\right)$ and is very close to -0.82 reported by ${ }_{523}$ Jardani et al. (2007). The obtained constant $A_{1}=-14.2$ deviates largely 524 from value of -9.23 proposed by Jardani et al. (2007). The reason is that ${ }_{525}$ $A_{1}$ is mainly dependent of chemical and interface parameters (Guarracino ${ }_{526}$ 
\& Jougnot, 2018). Therefore, it varies with mineral composition of rocks, ${ }_{527}$ electrolyte concentration, types of electrolyte etc.

media in which the minimum pore radius is 100 times the Debye length, ${ }_{531}$
that is around $1 \mu \mathrm{m}$ under environmental conditions. The porous medium ${ }_{532}$ is conceptualized as a bundle of tortuous capillary tubes with a pore-size ${ }_{533}$ distribution following a fractal law. The obtained model is linked to the ${ }_{534}$ applied voltage, back pressure, water saturation, irreducible water satura 535 tion and microstructural parameters of porous materials $\left(D_{f}, \varphi, \alpha, r_{\max }, \tau\right){ }_{536}$ From the model, the expressions for the electroosmosis pressure coefficient, ${ }_{537}$ the relative electroosmosis pressure coefficient, the maximum back pressure, ${ }_{38}$ the maximum flow rate, the flow rate-applied back pressure relation and the ${ }_{539}$ product of the permeability and formation factor of porous media are also ${ }_{540}$ obtained. To validate the model, the sensitivity of the relative electroosmosis ${ }_{541}$ pressure coefficient $K_{E}{ }^{r}$ to $S_{i r r}, \alpha$ and $D_{f}$ is analyzed and explained. The ${ }_{542}$ model predictions are then compared with published data in both cases of ${ }_{543}$ full saturation and partial saturation. The comparisons show that our model ${ }_{544}$ is able to explain well experimental data. From $K_{E}{ }^{r}$, the expression for the ${ }_{545}$ relative streaming potential coefficient $K_{S}{ }^{r}$ is also deduced. From that, the ${ }_{546}$ variation of $K_{S}{ }^{r}$ with the water saturation is predicted and compared with ${ }_{547}$ another model as well as experimental data from the dolomile rock sample. ${ }_{548}$ Addtionally, we also obtain an expression for the effective excess charge $\operatorname{den}_{549} \operatorname{sity} \widehat{Q}_{v}$. We find a good agreement between those obtained expressions and ${ }_{550}$ published data. We believe that the model proposed in this study can open ${ }_{551}$ up to new studies and modelling regarding electroosmotic phenomena and ${ }_{552}$ paving the way to to the development of new applications and technical devel ${ }_{553}$ opment in various disciplines from contaminated porous media remediation ${ }_{554}$ to masonery structures dewatering.

\section{Acknowledgments}

This research is funded by Vietnam National Foundation for Science ${ }_{557}$ and Technology Development (NAFOSTED) under grant number $103.99_{558}$ 2019.316. Additionally, D. Jougnot and A. Mendieta strongly thank the ${ }_{559}$ 
financial support of ANR EXCITING (grant ANR-17-CE06-0012) for the ${ }_{560} \mathrm{PhD}$ thesis funding of A. Mendieta.

\section{References}

Bandopadhyay, A., DasGupta, D., Mitra, S. K., \& Chakraborty, S. (2013). ${ }_{563}$ Electro-osmotic flows through topographically complicated porous media: 564 Role of electropermeability tensor. Phys. Rev. E, 87, 033006.

Bernabe, Y., \& Revil, A. (1995). Pore-scale heterogeneity, energy dissipation and the transport properties of rocks. Geophysical Research Letters, 22, 567 1529-1532.

${ }_{568}$ Bertolini, L., Coppola, L., Gastaldi, M., \& Redaelli, E. (2009). Electroos ${ }_{569}$ motic transport in porous construction materials and dehumidification of 570 masonry. Construction and Building Materials, 23, 254 - 263.

${ }_{571}$ Bol’eve, A., Crespy, A., Revil, A., Janod, F., \& Mattiuzzo, J. L. (2007). 572 Streaming potentials of granular media: Influence of the dukhin and ${ }_{573}$ reynolds numbers. Journal of Geophysical Research, B08204.

${ }_{574}$ Bruell, C. J., Segall, B. A., \& Walsh, M. T. (1992). Electroosomotic removal ${ }_{575}$ of gasoline hydrocarbons and tce from clay. Journal of Environmental 576 Engineering, 118, 68-83.

${ }_{577}$ Bruus, H. (2008). Theoretical Microfluidics. Oxford University Press; 1578 edition.

579 Cai, J. C., Hu, X. Y., Standnes, D. C., \& You, L. J. (2012a). An analytical 580 model for spontaneous imbibition in fractal porous media including gravity. 581 Colloids and Surfaces, A: Physicocemical and Engineering Aspects, 414, 582 233.

${ }_{583}$ Cai, J. C., You, L. J., Hu, X. Y., Wang, J., \& Peng, R. H. (2012b). Pre ${ }_{584}$ diction of effective permeability in porous media based on spontaneous 585 imbibition effect. International Journal of Modern Physics C, 23, DOI: ${ }_{586}$ 10.1142/S0129183112500544.

${ }_{587}$ Casagrande, L. (1983). Stabilization of soils by means of electroosmotic ${ }_{588}$ state-of-art. Journal of Boston Society of Civil Engineering, ASCE, 69, 589 255-302. 
${ }_{590}$ Cherubini, A., Garcia, B., Cerepi, A., \& Revil, A. (2018). Streaming poten ${ }_{591}$ tial coupling coefficient and transport properties of unsaturated carbonate ${ }_{59}$ rocks. Vadose Zone Journal, 17, 180030.

${ }_{593}$ Davis, J., James, R., \& Leckie, J. (1978). Surface ionization and complexas94tion at the oxide/water interface. i. computation of electrical double layer ${ }_{595}$ properties in simple electrolytes. Journal of Colloid and Interface Science, $596 \quad 63$.

${ }_{597}$ Feder, J., \& Aharony, A. (1989). Fractals in Physics. North Holland, Ams 598 terdam.

${ }_{599}$ Friedman, S. P. (2005). Soil properties influencing apparent electrical con 600 ductivity: a review. Computers and Electronics in Agriculture, 46, 45 - 60170.

${ }_{602}$ Ghanbarian, B., Hunt, A., P. Ewing, R., \& Sahimi, M. (2013). Tortuosity in 603 porous media: A critical review. Soil Science Society of America Journal, $604 \quad 77$, 1461-1477.

${ }_{605}$ Gierst, L. (1966). Double layer and electrode kinetics. Journal of the Amer ${ }_{006}$ ican Chemical Society, 88, 4768-4768.

${ }_{607}$ Glover, P., I. Zadjali, I., \& A Frew, K. (2006). Permeability prediction from 608 micp and nmr data using an electrokinetic approach. Geophysics, 71, 60 F49-F60.

${ }_{610}$ Glover, P. W. J., \& D'ery, N. (2010). Streaming potential coupling coefficient ${ }_{611}$ of quartz glass bead packs: Dependence on grain diameter, pore size, and ${ }_{612}$ pore throat radius. Geophysics, 75, F225-F241.

${ }_{613}$ Glover, P. W. J., \& Walker, E. (2009). Grain-size to effective pore-size ${ }_{614}$ transformation derived from electrokinetic theory. Geophysics, 74(1), E17$615 \quad$ E29.

616 Good, B. T., Bowman, C. N., \& Davis, R. H. (2006). An effervescent reaction ${ }_{617}$ micropump for portable microfluidic systems. Lab Chip, 6, 659-666.

618 Guarracino, L., \& Jougnot, D. (2018). A physically based analytical model to 619 describe effective excess charge for streaming potential generation in water ${ }_{220} \quad$ saturated porous media. Journal of Geophysical Research: Solid Earth, ${ }_{621} \quad 123,52-65$. 
${ }_{622}$ Han, S.-J., Kim, S.-S., \& Kim, B.-I. (2004). Electroosmosis and pore pressure ${ }_{623}$ development characteristics in lead contaminated soil during electrokinetic ${ }_{624}$ remediation. Geosciences Journal, 8, 85.

${ }_{622} \mathrm{Hu}, \mathrm{G}$., \& Li, D. (2007). Multiscale phenomena in microfluidics and nanoflu ${ }_{626}$ idics. Chemical Engineering Science, 62, 3443 - 3454.

${ }_{627} \mathrm{Hu}, \mathrm{X} ., \mathrm{Hu}, \mathrm{S}$., Jin, F., \& Huang, S. (2017). Physics of Petroleum Reservoirs.

${ }_{628}$ Springer-Verlag Berlin Heidelberg.

${ }_{629}$ Hunter, R. J. (1981). Zeta Potential in Colloid Science. Academic, New 630 York.

${ }_{631}$ Israelachvili, J. (1992). Intermolecular and Surface Forces. Academic Press.

${ }_{632}$ Jaafar, M. Z., Vinogradov, J., \& Jackson, M. D. (2009). Measure ${ }_{63}$ ment of streaming potential coupling coefficient in sandstones satu ${ }_{634}$ rated with high salinity nacl brine. Geophysical Research Letters, 36, ${ }_{635}$ doi:10.1029/2009GL040549.

${ }_{636}$ Jacob, H. M., \& Subirm, B. (2006). Electrokinetic and Colloid Transport ${ }_{637}$ Phenomena. Wiley-Interscience.

${ }_{638}$ Jardani, A., Revil, A., Boleve, A., Crespy, A., Dupont, J.-P., Barrash, W., \& 639 Malama, B. (2007). Tomography of the darcy velocity from self-potential 640 measurements. Geophysical Research Letters, 34.

${ }_{644}$ Jougnot, D., Linde, N., Revil, A., \& Doussan, C. (2012). Derivation of ${ }_{642}$ soilspecific streaming potential electrical parameters from hydrodynamic ${ }_{643}$ characteristics of partially saturated soils. Vadose Zone Journal, 11, 272- ${ }_{644} 286$.

${ }_{645}$ Jougnot, D., Mendieta, A., Leroy, P., \& Maineult, A. (2019). Exploring the ${ }_{646}$ effect of the pore size distribution on the streaming potential generation in ${ }_{647}$ saturated porous media, insight from pore network simulations. Journal ${ }_{648} \mathrm{O}$ Geophysical Research: Solid Earth, 124, 5315-5335.

${ }_{649}$ Jurin, J. (1719). Ii. an account of some experiments shown before the royal ${ }_{650}$ society; with an enquiry into the cause of the ascent and suspension of ${ }_{651}$ water in capillary tubes. Philosophical Transactions of the Royal Society ${ }_{65}$ of London, 30, 739-747. 
${ }_{653}$ Katz, A. J., \& Thompson, A. H. (1985). Fractal sandstone pores: Implica ${ }_{654}$ tions for conductivity and pore formation. Phys. Rev. Lett., 54, 1325-1328.

${ }_{655}$ Kirby, B. (2010). Micro and Nanoscale Fluid Mechanics: Transport in Mi656 crofluidic Devices. Cambridge University Press.

${ }_{657}$ Larue, O., Wakeman, R., Tarleton, E., \& Vorobiev, E. (2006). Pressure elec 658 troosmotic dewatering with continuous removal of electrolysis products.

659 Chemical Engineering Science, 61, 4732 - 4740.

${ }_{660}$ Leroy, P., \& Maineult, A. (2018). Exploring the electrical potential inside 661 cylinders beyond the DebyeHckel approximation: a computer code to solve ${ }_{62}$ the PoissonBoltzmann equation for multivalent electrolytes. Geophysical ${ }_{663}$ Journal International, 214, 58-69.

${ }_{664}$ Leroy, P., \& Revil, A. (2004). A triple-layer model of the surface electrochem 655 ical properties of clay minerals. Journal of Colloid and Interface Science, ${ }_{666} 270$, $371-380$.

${ }_{667}$ Levine, S., Marriott, J., Neale, G., \& Epstein, N. (1975). Theory of electroki ${ }_{668}$ netic flow in fine cylindrical capillaries at high zeta-potentials. Journal of 669 Colloid and Interface Science, 52, 136 - 149.

${ }_{670} \mathrm{Li}$, S. X., Pengra, D. B., \& P.Z.Wong (1995). Onsager's reciprocal relation ${ }_{671}$ and the hydraulic permeability of porous media. Physical Review E, 51, $6725748-$ 5751.

${ }_{673}$ Liang, M., Yang, S., Miao, T., \& Yu, B. (2015). Analysis of electroosmotic 674 characters in fractal porous media. Chemical Engineering Science, 127.

675 Liang, M., Yang, S., \& Yu, B. (2014). A fractal streaming current model for 676 charged microscale porous media. Journal of Electrostatics, 72.

${ }_{677}$ Linan Jiang, Mikkelsen, J., Jae-Mo Koo, Huber, D., Shuhuai Yao, Lian 67 Zhang, Peng Zhou, Maveety, J. G., Prasher, R., Santiago, J. G., Kenny, 679 T. W., \& Goodson, K. E. (2002). Closed-loop electroosmotic microchannel 680 cooling system for vlsi circuits. IEEE Transactions on Components and ${ }_{681}$ Packaging Technologies, 25, 347-355.

${ }_{682}$ Linde, N., Binley, A., Tryggvason, A., Pedersen, L. B., \& Revil, A. (2006). ${ }_{683}$ Improved hydrogeophysical characterization using joint inversion of cross684 
hole electrical resistance and ground-penetrating radar traveltime data. 685 Water Resources Research, 42.

${ }_{686}$ Lockhart, N., \& Hart, G. (1988). Electro-osmotic dewatering of fine suspen 687 sions: the efficacy of current interruptions. Drying Technology, 6, 415-423.

${ }_{688}$ Luong, D. T., \& Sprik, R. (2013). Streaming potential and electroosmosis 689 measurements to characterize porous materials. ISRN Geophysics, Article ${ }_{60} I D$ 496352, 8 pages.

${ }_{69}$ Lyklema, J. (1995). Fundamentals of Interface and Colloid Science. Aca692 demic Press.

${ }_{693}$ Mandelbrot, B. B. (1982). The Fractal Geometry of Nature. W.H. Freeman, 694 New York.

${ }_{695}$ Mohiuddin Mala, G., Li, D.-D., Werner, C., Jacobasch, H.-J., \& Ning, Y. ${ }_{69}$ (1997). Flow characteristics of water through a microchannel between two ${ }_{69}$ parallel plates with electrokinetic effects. International Journal of Heat ${ }_{998}$ and Fluid Flow, $18,489496$.

${ }_{699}$ Nourbehecht, B. (1963). Irreversible thermodynamic effects in inhomoge ${ }_{700}$ neous media and their applications in certain geoelectric problems. $\mathrm{PhD}$ 701 thesis, MIT Press, Cambridge, Mass, USA.

${ }_{702}$ Ohshima, H., \& Kondo, T. (1990). Electrokinetic flow between two parallel 703 plates with surface charge layers: Electro-osmosis and streaming potential. 704 Journal of Colloid and Interface Science, 135, 443-448.

705 Olivares, W., Croxton, T. L., \& McQuarrie, D. A. (1980). Electrokinetic flow 706 in a narrow cylindrical capillary. The Journal of Physical Chemistry, 84, 707 867-869.

${ }_{708}$ Ottosen, L., \& Ro"rig-Dalgaard, I. (2006). Drying brick masonry by electro 709 osmosis. In Proceedings of the Seventh International Masonry Conference. 710 British Masonry Society.

${ }_{711}$ Paillat, T., Moreau, E., P.O.Grimaud, \& Touchard, G. (2000). Electroki ${ }_{172}$ netic phenomena in porous media applied to soil decontamination. IEEE 713 Transactions on Dielectrics and Electrical Insulation, 7, 693-704. 
${ }_{714}$ Pascal, J., Oyanader, M., \& Arce, P. (2012). Effect of capillary geometry on ${ }_{715}$ predicting electroosmotic volumetric flowrates in porous or fibrous media. 716 Journal of Colloid and Interface Science, 378, 241 - 250.

717 Pengra, D., Li, S. X., \& Wong, P. (1999). Determination of rock properties 718 by low frequency ac electrokinetics. Journal of Geophysical Research, 104, 719 29485-29508.

${ }_{720}$ Pride, S. (1994). Governing equations for the coupled electromagnetics and 721 acoustics of porous media. Physical Review B, 50, 15678-15696.

722 Pride, S. R., \& Morgan, F. D. (1991). Electrokinetic dissipation induced by 723 seismic waves. Geophysics, 56, 914-925.

${ }_{724}$ Quincke, G. (1861). Ueber die fortfhrung materieller theilchen durch $\operatorname{str}_{725}$ mende elektricitt. Annalen der Physik, 189, 513-598.

${ }_{726}$ Reddy, K. R., Parupudi, U. S., Devulapalli, S. N., \& Xu, C. Y. (1997). Effects ${ }_{727}$ of soil composition on the removal of chromium by electrokinetics. Journal 728 of Hazardous Materials, 55, 135 - 158.

${ }_{722}$ Reuss, F. (1809). Sur un nouvel effet de l'lectricit galvanique. Mmoires de ${ }_{730} l a$ Societ Imperiale de Naturalistes de Moscou, 2, 327-336.

${ }_{731}$ Revil, A., Cathles III, L. M., \& Manhardt, P. D. (1999). Permeability of ${ }_{732}$ shaly sands. Water Resources Research, 3, 651-662.

${ }_{733}$ Revil, A., \& Leroy, P. (2004). Constitutive equations for ionic transport in ${ }_{734}$ porous shales. Journal of Geophysical Research: Solid Earth, 109. B03208.

${ }_{735}$ Revil, A., \& Linde, N. (2006). Chemico-electromechanical coupling in $\mathrm{mi}_{36}$ croporous media. Journal of Colloid and Interface Science, 302, 682 - ${ }_{37} 694$.

${ }_{738}$ Revil, A., Linde, N., Cerepi, A., Jougnot, D., Matthi, S., \& Finsterle, S. ${ }_{79}$ (2007). Electrokinetic coupling in unsaturated porous media. Journal of ${ }_{740}$ Colloid and Interface Science, 313, 315 - 327.

${ }_{744}$ Rice, C., \& Whitehead, R. (1965). Electrokinetic flow in a narrow cylindrical 742 capillary. J. Phys. Chem., 69, 4017-4024.

${ }_{743}$ Sen, P. N., \& Goode, P. A. (1992). Influence of temperature on electrical 744 conductivity on shaly sands. Geophysics, 57, 89-96. 
745 Singhal, V., Garimella, S. V., \& Raman, A. (2004). Microscale pumping tech ${ }_{746}$ nologies for microchannel cooling systems. Birck and NCN Publications, 74757.

${ }_{748}$ Smoluchowski, M. (1902). Contribution 'a la th'eorie de l'endosmose 'electrique ${ }_{749} \mathrm{et}$ de quelques ph'enom`enes corr'elatifs. Bulletin de l'Acad'emie des Sciences 750 de Cracovie, 8, 182-200.

${ }_{751}$ Soldi, M., Guarracino, L., \& Jougnot, D. (2019). An analytical effective ${ }_{752}$ excess charge density model to predict the streaming potential generated 753 by unsaturated flow. Geophysical Journal International, 216, 380-394.

${ }_{754}$ Thanh, L., \& Sprik, R. (2015). Zeta potential measurement using stream 75 ing potential in porous media. VNU Journal of Science: Mathematics ${ }_{756}$ Physics, 31, 56-65.

${ }_{757}$ Thanh, L. D., Jougnot, D., Van Do, P., \& Van Nghia A, N. (2019). A 758 physically based model for the electrical conductivity of water-saturated ${ }_{75}$ porous media. Geophysical Journal International, 219, 866-876.

${ }_{760}$ Thanh, L. D., Van Do, P., Van Nghia, N., \& Ca, N. X. (2018). A fractal ${ }_{761}$ model for streaming potential coefficient in porous media. Geophysical ${ }_{62}$ Prospecting, 66, 753-766.

763 Tsai, N.-C., \& Sue, C.-Y. (2007). Review of mems-based drug delivery and 764 dosing systems. Sensors and Actuators A: Physical, 134, 555 - 564.

${ }_{765}$ Vennela, N., Bhattacharjee, S., \& De, S. (2011). Sherwood number in porous 766 microtube due to combined pressure and electroosmotically driven flow. ${ }_{767}$ Chemical Engineering Science, 66, 6515 - 6524.

${ }_{768}$ Vinogradov, J., Jaafar, M. Z., \& Jackson, M. D. (2010). Measurement of 769 streaming potential coupling coefficient in sandstones saturated with nat ${ }_{700}$ ural and artificial brines at high salinity. Journal of Geophysical Research, 771 115, doi:10.1029/2010JB007593.

${ }_{772}$ Wang, J., Hu, H., \& Guan, W. (2016). The evaluation of rock permeability 773 with streaming current measurements. Geophysical Journal International, 774206 , 1563-1573.

${ }_{775}$ Wang, J., Hu, H., Guan, W., \& Li, H. (2015). Electrokinetic experimental 776 study on saturated rock samples: zeta potential and surface conductance. 777 Geophysical Journal International, 201, 869-877. 

6, 145-162.

${ }_{781}$ Wise, D. L., \& Trantolo, D. J. (1994). Remediation of Hazardous Waste ${ }_{782}$ Contaminated Soils. CRC Press.

${ }_{783} \mathrm{Wu}$, R. C., \& Papadopoulos, K. D. (2000). Electroosmotic flow through 784 porous media: cylindrical and annular models. Colloids and Surfaces A: ${ }_{785}$ Physicochemical and Engineering Aspects, 161, 469 - 476.

${ }_{786}$ Wyllie, M. R. J., \& Rose, W. (1950). Some theoretical considerations related ${ }_{787}$ to the quantitative evaluation of the physical characteristics of reservoir 788 rock from electrical log data. Society of Petroleum Engineers, .

${ }_{789}$ Yao, S., \& Santiago, J. G. (2003). Porous glass electroosmotic pumps: theory. 790 J. Colloid Interface Sci, 268, 133-142.

791 Yu, B., \& Cheng, P. (2002). A fractal permeability model for bi-dispersed 792 porous media. International Journal of Heat and Mass Transfer, 45, 2983- 793 2993.

${ }_{794} \mathrm{Yu}$, B., Lee, L. J., \& Cao, H. (2001). Fractal characters of pore microstruc ${ }_{795}$ tures of textile fabrics. Fractals, 09, 155-163.

796 Zeng, S., Chen, C. H., Mikkelsen, J. C., \& Santiago, J. G. (2001). Fabrication 797 and characterization of electroosmotic micropumps. Sens. Actuators B, 79, $798107-$ 114. 